\title{
UNIQUENESS THEOREMS IN INVERSE SPECTRAL THEORY FOR ONE-DIMENSIONAL SCHRÖDINGER OPERATORS
}

\author{
F. GESZTESY AND B. SIMON
}

\begin{abstract}
New unique characterization results for the potential $V(x)$ in connection with Schrödinger operators on $\mathbb{R}$ and on the half-line $[0, \infty)$ are proven in terms of appropriate Krein spectral shift functions. Particular results obtained include a generalization of a well-known uniqueness theorem of Borg and Marchenko for Schrödinger operators on the half-line with purely discrete spectra to arbitrary spectral types and a new uniqueness result for Schrödinger operators with confining potentials on the entire real line.
\end{abstract}

\section{INTRODUCTION}

The purpose of this article is to prove a variety of new uniqueness theorems for potentials $V(x)$ in one-dimensional Schrödinger operators $-\frac{d^{2}}{d x^{2}}+V$ on $\mathbb{R}$ and on the half-line $\mathbb{R}_{+}=[0, \infty)$ in terms of appropriate Krein spectral shift functions recently introduced in a series of papers describing new trace formulas for $V(x)$ on $\mathbb{R}[15],[17],[19],[20]$ and on $\mathbb{R}_{+}[14]$.

First we briefly recall these trace formulas for Schrödinger operators $H=-\frac{d^{2}}{d x^{2}}+$ $V$ on the real line $\mathbb{R}$ assuming $V$ to be real-valued, continuous, and bounded from below. In addition to $H$, one also considers the family of operators $H_{y}^{\beta}=-\frac{d^{2}}{d x^{2}}+V$, $\beta \in \mathbb{R} \cup\{\infty\}, y \in \mathbb{R}$, with an additional boundary condition of the type $g^{\prime}\left(y_{ \pm}\right)+$ $\beta g\left(y_{ \pm}\right)=0$ for elements $g$ in the domain of $H_{y}^{\beta}$; see (A.30) and (3.2) for detailed domain descriptions. Here, in obvious notation, $\beta=\infty$ denotes the corresponding operator $H_{y}^{\infty}$ with an additional Dirichlet boundary condition at $y \in \mathbb{R}$. Denoting by $\xi^{\beta}(\lambda, y)$ Krein's spectral shift function for the pair $\left(H_{y}^{\beta}, H\right), \beta \in \mathbb{R} \cup\{\infty\}, y \in \mathbb{R}$ (see (3.12)-(3.18)), the following trace formulas have been derived in [15] in the Dirichlet case $\beta=\infty$ and in $[20]$ for $\beta \in \mathbb{R}$ :

$$
\begin{aligned}
V(x)=E_{0}+\lim _{z \rightarrow i \infty} \int_{E_{0}}^{\infty} d \lambda \frac{z^{2}}{(\lambda-z)^{2}}\left[1-2 \xi^{\infty}(\lambda, x)\right], \\
E_{0}=\inf \{\sigma(H)\}, \beta=\infty, x \in \mathbb{R},
\end{aligned}
$$

Received by the editors February 27, 1995.

1991 Mathematics Subject Classification. Primary 34B24, 34L05, 81Q10; Secondary 34B20, $47 \mathrm{~A} 10$.

Key words and phrases. Schrödinger operators, inverse spectral theory, Krein's spectral shift function.

This material is based upon work supported by the National Science Foundation under Grant No. DMS-9101715. The U.S. Government has certain rights in this material. 


$$
\begin{array}{r}
V(x)=2 \beta^{2}+E_{0}^{\beta}(x)+\lim _{z \rightarrow i \infty} \int_{E_{0}^{\beta}(x)}^{\infty} d \lambda \frac{z^{2}}{(\lambda-z)^{2}}\left[1+2 \xi^{\beta}(\lambda, x)\right], \\
\left.E_{0}^{\beta}(x)=\inf \left\{\sigma H_{x}^{\beta}\right)\right\}, \beta \in \mathbb{R}, x \in \mathbb{R} .
\end{array}
$$

(Here $\sigma(\cdot)$ denotes the spectrum.) These trace formulas extend previous results by $[7-9],[12],[22],[26],[28],[29],[34],[35],[39],[40]$ in the short-range, periodic, and certain almost periodic cases.

A similar result can be derived for half-line Schrödinger operators. Assuming again $V$ to be real-valued, continuous, and bounded from below, denote by $H_{+, \alpha}=$ $-\frac{d^{2}}{d x^{2}}+V, \alpha \in[0, \pi)$, the family of Schrödinger operators on the half-line $\mathbb{R}_{+}=$ $[0, \infty)$ with the boundary condition $\sin (\alpha) g^{\prime}\left(0_{+}\right)+\cos (\alpha) g\left(0_{+}\right)=0$ for elements $g$ in the domain of $H_{+, \alpha}$ (cf. (A.14)). For $\alpha_{1}, \alpha_{2} \in(0, \pi), \alpha_{1} \neq \alpha_{2}$, let $\xi_{\alpha_{1}, \alpha_{2}}(\lambda)$ be Krein's spectral shift function for the pair $\left(H_{+, \alpha_{2}}, H_{+, \alpha_{1}}\right)$ (cf. (2.8)-(2.10)). Then the following trace formula can be inferred from the results in [14]:

$$
V(0)=\cot ^{2}(\alpha)+\lim _{z \rightarrow i \infty}\left\{-z-i \cot (\alpha) z^{1 / 2}+2 \int_{\mathbb{R}} d \lambda \frac{z^{2}}{(\lambda-z)^{2}} \xi_{0, \alpha}(\lambda)\right\}, \quad \alpha \in(0, \pi) .
$$

A quick look at (1.1), (1.2), and (1.3) reveals the fact that $\xi^{\beta}(\lambda, x), \lambda, x \in \mathbb{R}$, determines $V(x), x \in \mathbb{R}$, and $\xi_{0, \alpha}(\lambda), \lambda \in \mathbb{R}$, determines $V(0)$ in the half-line case. However, clearly both of these statements describe a mismatch and hence miss the point: $\xi^{\beta}(\lambda, x)$ depends on two real variables as opposed to one in $V(x)$ and, analogously, $\xi_{0, \alpha}(\lambda)$ depends on one real variable while $V(0)$ is just a constant. From the point of view of inverse spectral theory, the problems that need clarification appear to be the following: Does $\xi^{\beta}\left(\lambda, x_{0}\right)$ for fixed $x_{0} \in \mathbb{R}$ and all $\lambda \in \mathbb{R}$ determine $V(x)$ for all $x \in \mathbb{R}$ and, similarly, does $\xi_{\alpha_{1}, \alpha_{2}}(\lambda), \alpha_{1} \neq \alpha_{2}$, for all $\lambda \in \mathbb{R}$ determine $V(x)$ for all $x \geq 0$ in the half-line case? The present paper provides complete solutions to these problems.

In Section 2 we treat the half-line case and provide an affirmative answer to the problem posed: $\xi_{\alpha_{1}, \alpha_{2}}(\lambda), \alpha_{1} \neq \alpha_{2}$, for a.e. $\lambda \in \mathbb{R}$ indeed uniquely determines $V(x)$ for a.e. $x \geq 0$ (cf. Theorem 2.4), extending a well-known result of Borg [5] and Marchenko [32], obtained independently from each other around 1952 for operators with purely discrete spectrum, to arbitrary spectral types (see Corollary 2.5). We conclude Section 2 with an application of our main Theorem 2.4 to threedimensional Schrödinger operators with spherically symmetric potentials, and state a new uniqueness theorem in this context (cf. Theorem 2.6).

Section 3 is devoted to Schrödinger operators on the entire real line. While the corresponding question posed concerning $\xi^{\beta}\left(\lambda, x_{0}\right)$ turns out to have a negative answer, that is, $\xi^{\beta}\left(\lambda, x_{0}\right)$ for fixed $x_{0} \in \mathbb{R}$ and a.e. $\lambda \in \mathbb{R}$ in general cannot determine $V$ uniquely for a.e. $x \in \mathbb{R}$, Theorem 3.2 shows that $\xi^{\beta_{1}}\left(\lambda, x_{0}\right)$ and $\xi^{\beta_{2}}\left(\lambda, x_{0}\right), \beta_{1} \neq$ $\beta_{2}$, for a.e. $\lambda \in \mathbb{R}$ uniquely determine $V$ a.e. except in the Dirichlet and Neumann cases $\beta_{1}=0, \beta_{2}=\infty$, respectively, $\beta_{1}=\infty, \beta_{2}=0$. In the latter case, $V$ is uniquely determined up to reflection symmetry with respect to $x_{0}$. When combining $\xi^{\beta}\left(\lambda, x_{0}\right), \lambda \in \mathbb{R}$, with additional Dirichlet data and/or norming constants, further unique characterizations of $V$ can be achieved. This is illustrated in connection with 
Theorem 3.6, which provides a new uniqueness result for Schrödinger operators on $\mathbb{R}$ with purely discrete spectra.

Since our techniques rely heavily on the use of certain properties of Herglotz functions and especially on the Weyl-Titchmarsh theory, we collected a variety of pertinent results in Appendix A.

Perhaps we should emphasize at this point that we do not discuss explicit reconstruction procedures for $V(x)$ in this paper (the reader can find standard results on reconstruction techniques, e.g., in [13],[29],[30],[32], and [33]). Here we exclusively focus on deriving new minimal sets of spectral data which uniquely determine the potential $V$ a.e. The basic outline of our philosophy of how to recover $V(x)$ from $\xi^{\infty}\left(\lambda, x_{0}\right), \lambda \in \mathbb{R}$, and Dirichlet data is described in [15]. We shall return to this topic elsewhere.

Analogous results for second-order finite difference operators are in preparation [18].

\section{SchrÖdinger operators on $[\mathbf{0}, \infty)$}

In this section we shall describe a uniqueness result for Schrödinger operators on the half-line $[0, \infty)$, which extends a well-known theorem of Borg [5] and Marchenko [32] in the special case of purely discrete spectra to arbitrary spectral types.

We shall freely exploit the notation introduced in Appendix A and recall $\tau_{+}$, $H_{+, \alpha}, \phi_{\alpha}, \theta_{\alpha}, \psi_{+, \alpha}, m_{+, \alpha}, d \rho_{+, \alpha}$, and $G_{+, \alpha}\left(z, x, x^{\prime}\right)$ as introduced in (A.13)-(A.27). In particular, we shall assume hypothesis (A.12), that is,

$$
V \in L^{1}([0, R]) \text { for all } R>0, \quad V \text { real-valued }
$$

throughout this section and recall that $H_{+, \alpha}$, defined in terms of separated boundary conditions, is a real operator of uniform spectral multiplicity one.

The basic uniqueness criterion for Schrödinger operators on the half-line $[0, \infty)$ we shall rely on repeatedly in the following can be stated as follows.

Theorem 2.1 (See, e.g., [32]). Suppose $\alpha_{1}, \alpha_{2} \in[0, \pi), \alpha_{1} \neq \alpha_{2}$, and define $H_{+, j, \alpha_{j}}, m_{+, j, \alpha_{j}}, \rho_{+, j, \alpha_{j}}$ associated with the differential expressions $\tau_{j}=-\frac{d^{2}}{d x^{2}}+$ $V_{j}(x), x \geq 0$, where $V_{j}, j=1,2$, satisfy hypothesis (2.1). Then the following are equivalent:

(i) $m_{+, 1, \alpha_{1}}(z)=m_{+, 2, \alpha_{2}}(z), z \in \mathbb{C}_{+}$.

(ii) $\rho_{+, 1, \alpha_{1}}((-\infty, \lambda])=\rho_{+, 2, \alpha_{2}}((-\infty, \lambda]), \lambda \in \mathbb{R}$.

(iii) $\alpha_{1}=\alpha_{2}$ and $V_{1}(x)=V_{2}(x)$ for a.e. $x \geq 0$.

We begin our analysis with a simple warm-up relating Green's functions for different boundary conditions at $x=0$. (We also recall our convention of Appendix A to fix the boundary condition (if any) at $x=+\infty$.)

Lemma 2.2. Let $\alpha_{j} \in[0, \pi), j=1,2, x, x^{\prime} \in \mathbb{R}_{+}$, and $z \in \mathbb{C} \backslash\left\{\sigma\left(H_{+, \alpha_{1}}\right) \cup\right.$ $\left.\sigma\left(H_{+, \alpha_{2}}\right)\right\}$. Then

(i)

$$
G_{+, \alpha_{2}}\left(z, x, x^{\prime}\right)-G_{+, \alpha_{1}}\left(z, x, x^{\prime}\right)=-\frac{\psi_{+, \alpha_{1}}(z, x) \psi_{+, \alpha_{1}}\left(z, x^{\prime}\right)}{\cot \left(\alpha_{2}-\alpha_{1}\right)+m_{+, \alpha_{1}}(z)}
$$


(ii)

$$
\begin{aligned}
\frac{G_{+, \alpha_{2}}(z, 0,0)}{G_{+, \alpha_{1}}(z, 0,0)} & =\frac{1}{\left(\beta_{1}-\beta_{2}\right) \sin ^{2}\left(\alpha_{1}\right)\left[\cot \left(\alpha_{2}-\alpha_{1}\right)+m_{+, \alpha_{1}}(z)\right]} \\
& =\left(\beta_{1}-\beta_{2}\right) \sin ^{2}\left(\alpha_{2}\right)\left[\cot \left(\alpha_{2}-\alpha_{1}\right)-m_{+, \alpha_{2}}(z)\right], \\
\beta_{j} & =\cot \left(\alpha_{j}\right), \quad j=1,2 .
\end{aligned}
$$

$$
\begin{aligned}
\operatorname{Tr}\left[\left(H_{+, \alpha_{2}}-z\right)^{-1}-\left(H_{+, \alpha_{1}}-z\right)^{-1}\right] & =-\frac{d}{d z} \ln \left[\cot \left(\alpha_{2}-\alpha_{1}\right)+m_{+, \alpha_{1}}(z)\right] \\
& =\frac{d}{d z} \ln \left[\cot \left(\alpha_{2}-\alpha_{1}\right)-m_{+, \alpha_{2}}(z)\right] .
\end{aligned}
$$

Proof. (2.2) is a direct consequence of (A.16)-(A.18), (A.23), and (A.38). Similarly, (2.3) and (2.4) follow by combining (A.25) and (A.38). (2.5) follows from (2.2) and (A.44) in the limit $z_{1} \rightarrow z_{2}=z$. (2.6) is clear from

$$
\cot \left(\alpha_{2}-\alpha_{1}\right)+m_{+, \alpha_{1}}(z)=\left[\sin \left(\alpha_{2}-\alpha_{1}\right)\right]^{2}\left[\cot \left(\alpha_{2}-\alpha_{1}\right)-m_{+, \alpha_{2}}(z)\right]^{-1}
$$

a simple consequence of (A.38).

Since $m_{+, \alpha}(z)$ is a Herglotz function, we may now introduce Krein's spectral shift function [27] $\xi_{\alpha_{1}, \alpha_{2}}(\lambda)$ for the pair $\left(H_{+, \alpha_{2}}, H_{+, \alpha_{1}}\right)$ according to (A.2), (A.4) by

$$
\begin{aligned}
& \cot \left(\alpha_{2}-\alpha_{1}\right)+m_{+, \alpha_{1}}(z)=\exp \left\{\operatorname{Re}\left[\ln \left(\cot \left(\alpha_{2}-\alpha_{1}\right)+m_{+, \alpha_{1}}(i)\right)\right]\right. \\
& \left.\quad+\int_{\mathbb{R}}\left[\frac{1}{\lambda-z}-\frac{\lambda}{1+\lambda^{2}}\right] \xi_{\alpha_{1}, \alpha_{2}}(\lambda) d \lambda\right\}, \quad 0 \leq \alpha_{1}<\alpha_{2}<\pi, z \in \mathbb{C} \backslash \mathbb{R} .
\end{aligned}
$$

This is extended to all $\alpha_{1}, \alpha_{2} \in[0, \pi)$ by

$$
\xi_{\alpha, \alpha}(\lambda)=0, \quad \xi_{\alpha_{2}, \alpha_{1}}(\lambda)=-\xi_{\alpha_{1}, \alpha_{2}}(\lambda) \quad \text { for a.e. } \lambda \in \mathbb{R} .
$$

(2.7) then implies

$$
\begin{aligned}
& \cot \left(\alpha_{2}-\alpha_{1}\right)-m_{+, \alpha_{2}}(z)=\exp \left\{\operatorname{Re}\left[\ln \left(\cot \left(\alpha_{2}-\alpha_{1}\right)-m_{+, \alpha_{2}}(i)\right)\right]\right. \\
& \left.\quad-\int_{\mathbb{R}}\left[\frac{1}{\lambda-z}-\frac{\lambda}{1+\lambda^{2}}\right] \xi_{\alpha_{1}, \alpha_{2}}(\lambda) d \lambda\right\}, \quad 0 \leq \alpha_{1}<\alpha_{2}<\pi, z \in \mathbb{C} \backslash \mathbb{R} .
\end{aligned}
$$

Next we summarize a few properties of $\xi_{\alpha_{1}, \alpha_{2}}(\lambda)$. 
Lemma 2.3. (i) Suppose $0 \leq \alpha_{1}<\alpha_{2}<\pi$. Then for a.e. $\lambda \in \mathbb{R}$,

$$
\xi_{\alpha_{1}, \alpha_{2}}(\lambda)=\left\{\begin{array}{l}
\lim _{\epsilon \downarrow 0} \pi^{-1} \operatorname{Im}\left\{\ln \left[\cot \left(\alpha_{2}-\alpha_{1}\right)+m_{+, \alpha_{1}}(\lambda+i \epsilon)\right]\right\} \\
-\lim _{\epsilon \downarrow 0} \pi^{-1} \operatorname{Im}\left\{\ln \left[\cot \left(\alpha_{2}-\alpha_{1}\right)-m_{+, \alpha_{2}}(\lambda+i \epsilon)\right]\right\} \\
\lim _{\epsilon \downarrow 0} \pi^{-1} \operatorname{Im}\left\{\ln \left[\frac{1}{\sin \left(\alpha_{1}\right)} \frac{G_{+, \alpha_{1}}(\lambda+i \epsilon, 0,0)}{G_{+, \alpha_{2}}(\lambda+i \epsilon, 0,0)}\right]\right\} .
\end{array}\right.
$$

(For $\alpha_{1}=0, G_{+, \alpha_{1}}(\lambda+i \epsilon, 0,0) / \sin \left(\alpha_{1}\right)$ has to be replaced by -1 in (2.13) according to (A.25).) Moreover,

$$
0 \leq \xi_{\alpha_{1}, \alpha_{2}}(\lambda) \leq 1 \text { a.e. }
$$

(ii) Let $\alpha_{j} \in[0, \pi), 1 \leq j \leq 3$. Then the "chain rule"

$$
\xi_{\alpha_{1}, \alpha_{3}}(\lambda)=\xi_{\alpha_{1}, \alpha_{2}}(\lambda)+\xi_{\alpha_{2}, \alpha_{3}}(\lambda)
$$

holds for a.e. $\lambda \in \mathbb{R}$.

(iii) For all $\alpha_{1}, \alpha_{2} \in[0, \pi)$,

$$
\xi_{\alpha_{1}, \alpha_{2}} \in L^{1}\left(\mathbb{R} ;\left(1+\lambda^{2}\right)^{-1} d \lambda\right) .
$$

(iv) Assume $\alpha_{1}, \alpha_{2} \in[0, \pi), \alpha_{1} \neq \alpha_{2}$. Then

$$
\xi_{\alpha_{1}, \alpha_{2}} \in L^{1}\left(\mathbb{R} ;(1+|\lambda|)^{-1} d \lambda\right) \text { if and only if } \alpha_{1}, \alpha_{2} \in(0, \pi) .
$$

(v) For all $\alpha_{1}, \alpha_{2} \in[0, \pi)$,

$$
\operatorname{Tr}\left[\left(H_{+, \alpha_{2}}-z\right)^{-1}-\left(H_{+, \alpha_{1}}-z\right)^{-1}\right]=-\int_{\mathbb{R}}(\lambda-z)^{-2} \xi_{\alpha_{1}, \alpha_{2}}(\lambda) d \lambda .
$$

Proof. (i) (2.11)-(2.13) follow from (2.3), (2.4) (resp. (2.7)), (2.8), (A.2), and (A.4). (2.14) is clear from (A.4).

(ii) is a consequence of (2.13).

(iii) is obvious from $0 \leq\left|\xi_{\alpha_{1}, \alpha_{2}}\right| \leq 1$ a.e.

(iv) By (2.9) we may assume $0 \leq \alpha_{1}<\alpha_{2}<\pi$. Then (A.39) yields

$$
\cot \left(\alpha_{2}-\alpha_{1}\right)-m_{+, \alpha_{2}}(z) \underset{z \rightarrow i \infty}{=} \begin{cases}0, & \alpha_{1}=0, \\ \cot \left(\alpha_{2}-\alpha_{1}\right)-\cot \left(\alpha_{2}\right)>0, & 0<\alpha_{1}<\alpha_{2}<\pi,\end{cases}
$$

and it suffices to apply Theorem A.1(iii) to $\cot \left(\alpha_{2}-\alpha_{1}\right)-m_{+, \alpha_{2}}(z)$ taking into account (2.10).

(v) follows from (2.5) and from applying $-\frac{d}{d z} \ln (\cdot)$ to $(2.8)$.

We note that $\xi_{\alpha_{1}, \alpha_{2}}(\lambda)$ (for $\alpha_{1}, \alpha_{2} \in(0, \pi)$ ) has been introduced by Javrjan [23],[24]. In particular, he proved (2.5) and (2.18) in the non-Dirichlet cases where $0<\alpha_{1}, \alpha_{2}<\pi$. We also remark that (2.18) extends to more general situations of the type

$$
\operatorname{Tr}\left[F\left(H_{+, \alpha_{2}}\right)-F\left(H_{+, \alpha_{1}}\right)\right]=\int_{\mathbb{R}} F^{\prime}(\lambda) \xi_{\alpha_{1}, \alpha_{2}}(\lambda) d \lambda
$$

for appropriate functions $F$ (see, e.g., [38]).

Given these preliminaries, we are now able to state our main uniqueness result for half-line Schrödinger operators. 
Theorem 2.4. Suppose $V_{j}$ satisfy hypothesis (2.1), and introduce the differential expressions $\tau_{j}=-\frac{d^{2}}{d x^{2}}+V_{j}(x), x \geq 0, j=1,2$. Let $\alpha_{j, \ell} \in[0, \pi), \ell=1,2$, suppose $0 \leq \alpha_{1,1}<\alpha_{1,2}<\pi, 0 \leq \alpha_{2,1}<\alpha_{2,2}<\pi$, and define $H_{+, j, \alpha_{j, \ell}}$ for $j, \ell=1,2$ associated with $\tau_{j}$ as in (A.14). In addition, let $\xi_{j, \alpha_{j, 1}, \alpha_{j, 2}}, j=1,2$, be Krein's spectral shift function for the pair $\left(H_{+, j, \alpha_{j, 1}}, H_{+, j, \alpha_{j, 2}}\right)$. Then the following are equivalent:

(i) $\xi_{1, \alpha_{1,1}, \alpha_{1,2}}(\lambda)=\xi_{2, \alpha_{2,1}, \alpha_{2,2}}(\lambda)$ for a.e. $\lambda \in \mathbb{R}$.

(ii) $\alpha_{1,1}=\alpha_{2,1}, \alpha_{1,2}=\alpha_{2,2}$, and $V_{1}(x)=V_{2}(x)$ for a.e. $x \geq 0$.

Proof. We only need to prove that (i) implies (ii). From Lemma 2.3(iv), one infers that

$$
\alpha_{j, 1} \underset{(=)}{>} 0 \text { if and only if } \int_{\mathbb{R}}(1+|\lambda|)^{-1}\left|\xi_{\alpha_{j, 1}, \alpha_{j, 2}}(\lambda)\right| d \lambda \underset{(=)}{<\infty} \infty, \quad j=1,2 .
$$

Since by hypothesis $\alpha_{1,1} \underset{(=)}{>} 0$ if and only if $\alpha_{2,1} \underset{(=)}{>} 0$, one is led to the following case distinction.

a) $0<\alpha_{1,1}<\alpha_{1,2}<\pi, 0<\alpha_{2,1}<\alpha_{2,2}<\pi$. Then (2.10) and (A.39) imply

$$
\begin{gathered}
\int_{z}^{\infty} d z^{\prime} \int_{\mathbb{R}}\left(\lambda-z^{\prime}\right)^{-2} \xi_{j, \alpha_{j, 1}, \alpha_{j, 2}}(\lambda) d \lambda=\ln \left[\frac{\cot \left(\alpha_{j, 2}-\alpha_{j, 1}\right)-m_{+, j, \alpha_{j, 2}}(z)}{\cot \left(\alpha_{j, 2}-\alpha_{j, 1}\right)-\cot \left(\alpha_{j, 2}\right)}\right] \\
\underset{z \rightarrow i \infty}{=}\left(\beta_{j, 2}-\beta_{j, 1}\right) i z^{-1 / 2}+\left(\beta_{j, 1}^{2}-\beta_{j, 2}^{2}\right) 2^{-1} z^{-1}+o\left(z^{-1}\right), \\
\beta_{j, \ell}=\cot \left(\alpha_{j, \ell}\right), \quad j, \ell=1,2 .
\end{gathered}
$$

Given (i), the asymptotic behavior (2.23) then yields

$$
\alpha_{1,1}=\alpha_{2,1} \quad \text { and } \quad \alpha_{1,2}=\alpha_{2,2} .
$$

Insertion of (2.24) into (2.22), still assuming (i), then yields

$$
m_{+, 1, \alpha_{1,2}}(z)=m_{+, 2, \alpha_{1,2}}(z)
$$

and hence $V_{1}=V_{2}$ a.e. by Theorem 2.1.

b) $0=\alpha_{1,1}<\alpha_{1,2}<\pi, 0=\alpha_{2,1}<\alpha_{2,2}<\pi$. Then (2.10) and (A.39) imply

$$
\begin{aligned}
\int_{i}^{z} d z^{\prime} \int_{\mathbb{R}} & \left(\lambda-z^{\prime}\right)^{-2} \xi_{j, 0, \alpha_{j, 2}}(\lambda) d \lambda \\
= & -\ln \left[\frac{\cot \left(\alpha_{j, 2}\right)-m_{+, j, \alpha_{j, 2}}(z)}{\cot \left(\alpha_{j, 2}\right)-m_{+, j, \alpha_{j, 2}}(i)}\right] \\
= & \ln \left(z^{1 / 2}\right)+\ln \left[i \sin ^{2}\left(\alpha_{j, 2}\right)\right]+\ln \left[\cot \left(\alpha_{j, 2}\right)-m_{+, j, \alpha_{j, 2}}(i)\right] \\
& \quad-\cot \left(\alpha_{j, 2}\right) i z^{-1 / 2}+o\left(z^{-1 / 2}\right), \quad j=1,2 .
\end{aligned}
$$

Given (i), the $O\left(z^{-1 / 2}\right)$-term in (2.27) then yields

$$
\alpha_{1,2}=\alpha_{2,2}
$$


and the $O(1)$-term in (2.27) yields

$$
m_{+, 1, \alpha_{1,2}}(i)=m_{+, 2, \alpha_{1,2}}(i) .
$$

Inserting (2.28) and (2.29) into (2.26), still assuming (i), then yields

$$
m_{+, 1, \alpha_{1,2}}(z)=m_{+, 2, \alpha_{1,2}}(z)
$$

and hence again, $V_{1}=V_{2}$ a.e. by Theorem 2.1 .

As a corollary, we obtain a well-known uniqueness result originally due to Borg [5] and Marchenko [32], obtained independently in 1952.

Corollary 2.5 (Borg [5], Theorem 1; Marchenko [32], Theorem 2.3.2; see also [30]). Define $\tau_{j}$ and $H_{+, j, \alpha}, \alpha \in[0, \pi)$, as in Theorem 2.4. Assume in addition that $H_{+, 1, \alpha_{1}}$ and $H_{+, 2, \alpha_{2}}$ have purely discrete spectra for some (and hence for all) $\alpha_{j} \in[0, \pi)$, that is,

$$
\sigma_{\mathrm{ess}}\left(H_{+, j, \alpha_{j}}\right)=\emptyset \quad \text { for some } \alpha_{j} \in[0, \pi), j=1,2 .
$$

Then the following are equivalent:

(i) $\sigma\left(H_{+, 1, \alpha_{1,1}}\right)=\sigma\left(H_{+, 2, \alpha_{2,1}}\right), \sigma\left(H_{+, 1, \alpha_{1,2}}\right)=\sigma\left(H_{+, 2, \alpha_{2,2}}\right), \alpha_{j, \ell} \in[0, \pi), j, \ell=$ $1,2, \sin \left(\alpha_{1,1}-\alpha_{1,2}\right) \neq 0$.

(ii) $\alpha_{1,1}=\alpha_{2,1}, \alpha_{1,2}=\alpha_{2,2}$, and $V_{1}(x)=V_{2}(x)$ for a.e. $x \geq 0$.

Proof. Without loss of generality, we may assume $0 \leq \alpha_{1,1}<\alpha_{1,2}<\pi, 0 \leq \alpha_{2,1}<$ $\alpha_{2,2}<\pi$, and hence we need to prove that (i) implies $\xi_{1, \alpha_{1,1}, \alpha_{1,2}}=\xi_{2, \alpha_{2,1}, \alpha_{2,2}}$ a.e. First we note that $\xi_{j, \alpha_{j, 1}, \alpha_{j, 2}}(\lambda)$, being Krein's spectral shift function for the pair $\left(H_{+, j, \alpha_{j, 2}}, H_{+, j, \alpha_{j, 1}}\right), j=1,2$, increases (decreases) by 1 whenever $\lambda$ passes an eigenvalue of $H_{+, j, \alpha_{j, 1}}\left(H_{+, j, \alpha_{j, 2},}\right)$ as $\lambda$ increases from $-\infty$ to $+\infty$, and stays constant otherwise. (We recall that $\sigma\left(H_{+, \alpha}\right)$ is simple.) This step-function behavior, together with $0 \leq \xi_{j, \alpha_{j, 1}, \alpha_{j, 2}} \leq 1$ a.e., indeed yields $\xi_{1, \alpha_{1,1}, \alpha_{1,2}}=\xi_{2, \alpha_{2,1}, \alpha_{2,2}}$ a.e. and one can apply Theorem 2.4.

Roughly speaking, Corollary 2.5 says that two sets of purely discrete spectra $\sigma\left(H_{+, \alpha_{1}}\right), \sigma\left(H_{+, \alpha_{2}}\right)$ associated with distinct boundary conditions at $x=0$ (but a fixed boundary condition (if any) at $+\infty$ ), that is, $\sin \left(\alpha_{2}-\alpha_{1}\right) \neq 0$, uniquely determine $V$ a.e. Our main result, Theorem 2.4, removes all a priori spectral hypotheses and shows that Krein's spectral shift function $\xi_{\alpha_{1}, \alpha_{2}}(\lambda)$ for the pair $\left(H_{+, \alpha_{2}}, H_{+, \alpha_{1}}\right)$ with distinct boundary conditions at $x=0, \sin \left(\alpha_{2}-\alpha_{1}\right) \neq 0$, uniquely determines $V$ a.e. This illustrates that Theorem 2.4 is the natural generalization of Borg's and Marchenko's theorem from the discrete spectrum case to arbitrary spectral types.

Finally, we give a simple application of Theorem 2.4 in the context of threedimensional Schrödinger operators with spherically symmetric potentials.

Assuming hypothesis (2.1) for $V$, we introduce the potential

$$
v(x)=V(|x|), \quad x \in \mathbb{R}^{3},
$$

and define the self-adjoint Schrödinger operator $h$ in $L^{2}\left(\mathbb{R}^{3}\right)$ associated with the differential expression $-\Delta+v(x)$ by decomposition with respect to angular momenta, 
which represents $h$ as an infinite direct sum of half-line operators in $L^{2}\left(\mathbb{R}_{+} ; r^{2} d r\right)$ associated with differential expressions of the type

$$
\widehat{\tau}_{+, \ell}=-\frac{d^{2}}{d r^{2}}-\frac{2}{r} \frac{d}{d r}+\frac{\ell(\ell+1)}{r^{2}}+V(r), \quad r=|x|>0, \ell \in \mathbb{N}_{0}=\mathbb{N} \cup\{0\} .
$$

A simple unitary transformation reduces (2.33) to

$$
\tau_{+, \ell}=-\frac{d^{2}}{d r^{2}}+\frac{\ell(\ell+1)}{r^{2}}+V(r)
$$

and associated Hilbert space $L^{2}\left(\mathbb{R}_{+}\right)$(see, e.g., [37], Appendix to Sect. X.1).

Next, let $g\left(z, x, x^{\prime}\right), x \neq x^{\prime}$, denote the Green's function of $h$ (i.e., the integral kernel of $\left.(h-z)^{-1}\right)$ and define another self-adjoint operator $h_{\beta}$ in $L^{2}\left(\mathbb{R}^{3}\right)$ by

$$
\begin{aligned}
\left(h_{\beta}-z\right)^{-1}=(h-z)^{-1}+D_{\beta}(z)^{-1}(\overline{g(z, 0, \cdot)}, \cdot) g(z, \cdot, 0), & \\
\beta & \in \mathbb{R}, z \in \mathbb{C} \backslash\left\{\sigma\left(h_{\beta}\right) \cup \sigma(h)\right\},
\end{aligned}
$$

where

$$
D_{\beta}(z)=\beta-\lim _{|\epsilon| \downarrow 0}\left[g(z, 0, \epsilon)-(4 \pi|\epsilon|)^{-1}\right], \quad z \in \mathbb{C} \backslash \sigma(h) .
$$

As shown, for example, in [1],[41], $h_{\beta}$ models $h$ plus an additional point (delta) interaction centered at $x=0$ whose strength is parametrized by $\beta \in \mathbb{R}$. (Clearly, $h_{\infty}=h$.) The function $D_{\beta}(z)$ is Herglotz, and one computes (see [14])

$$
\operatorname{Tr}\left[\left(h_{\beta}-z\right)^{-1}-(h-z)^{-1}\right]=-\frac{d}{d z} \ln \left[D_{\beta}(z)\right] .
$$

This then allows one to define Krein's spectral shift function $\xi_{\beta}(\lambda)$ for the pair $\left(h_{\beta}, h\right)$ by

$$
\xi_{\beta}(\lambda)=\lim _{\epsilon \downarrow 0} \pi^{-1} \operatorname{Im}\left\{\ln \left[D_{\beta}(\lambda+i \epsilon)\right]\right\} \text { a.e. }
$$

which yields

$$
\operatorname{Tr}\left[\left(h_{\beta}-z\right)^{-1}-(h-z)^{-1}\right]=-\int_{\mathbb{R}}(\lambda-z)^{-2} \xi_{\beta}(\lambda) d \lambda .
$$

Our uniqueness result for three-dimensional Schrödinger operators then reads as follows.

Theorem 2.6. Define $h_{j}, h_{j, \beta_{j}}, \beta_{j} \in \mathbb{R}$, associated with $-\Delta+v_{j}(x), x \in \mathbb{R}^{3}$, $j=1,2$, and introduce Krein's spectral shift function $\xi_{j, \beta_{j}}(\lambda)$ for the pair $\left(h_{j, \beta_{j}}, h_{j}\right)$, $j=1,2$. Then the following are equivalent:

(i) $\xi_{1, \beta_{1}}(\lambda)=\xi_{2, \beta_{2}}(\lambda)$ for a.e. $\lambda \in \mathbb{R}$.

(ii) $\beta_{1}=\beta_{2}$ and $v_{1}(x)=v_{2}(x)$ for a.e. $x \in \mathbb{R}^{3}$. 
Proof. Since $\tau_{+, \ell}$ is l.p. at $r=0$ for all $\ell=\mathbb{N}$, the whole problem can be reduced to the angular momentum sector $\ell=0$. For $\ell=0$, however, $h$ corresponds to $H_{+, \infty}$ and $h_{\beta}$ to $H_{+, \alpha}, \beta=\cot (\alpha)$, in the notation of (A.14). In particular, $\xi_{\beta}(\lambda)$ introduced in (2.38) corresponds to $\xi_{0, \alpha}(\lambda)$ in our notation (2.8). Hence, an application of Theorem 2.4 completes the proof.

An analogous result could be derived for two-dimensional Schrödinger operators with centrally symmetric potentials. Since this requires the replacement of $\tau_{+}=$ $-\frac{d^{2}}{d x^{2}}+V(x), x \geq 0$, by

$$
\tau_{+}=-\frac{d^{2}}{d x^{2}}-\frac{1}{4 x^{2}}+V(x), \quad x>0,
$$

a differential expression singular at $x=0$, we omit further details at this point.

\section{SCHRÖDINGER OPERATORS ON $\mathbb{R}$}

This section explores uniqueness results for Schrödinger operators on the whole real line.

As in Section 2, we shall rely on the notation introduced in Appendix A and hence recall $\tau, H, \phi_{\alpha}, \theta_{\alpha}, \psi_{ \pm, \alpha}, m_{ \pm, \alpha}, d \rho_{ \pm, \alpha}$, and $G\left(z, x, x^{\prime}\right)$ as introduced in (A.29)-(A.47). In particular, we shall assume hypothesis (A.28), that is,

$$
V \in L_{\mathrm{loc}}^{1}(\mathbb{R}), \quad V \text { real-valued }
$$

throughout this section. Following [20], we introduce, in addition, the following family of self-adjoint operators $H_{y}^{\beta}$ in $L^{2}(\mathbb{R})$,

$$
\begin{gathered}
H_{y}^{\beta} f=\tau f, \quad \beta \in \mathbb{R} \cup\{\infty\}, \quad y \in \mathbb{R}, \\
\mathcal{D}\left(H_{y}^{\beta}\right)=\left\{g \in L ^ { 2 } \left(\mathbb{R} \mid g, g^{\prime} \in A C([y, \pm R]) \text { for all } R>0 ; g^{\prime}\left(y_{ \pm}\right)+\beta g\left(y_{ \pm}\right)=0 ;\right.\right. \\
\left.\lim _{R \rightarrow \pm \infty} W\left(f_{ \pm}\left(z_{ \pm}\right), g\right)(R)=0 ; \tau g \in L^{2}(\mathbb{R})\right\} .
\end{gathered}
$$

Thus $H_{y}^{D}:=H_{y}^{\infty}\left(H_{y}^{N}:=H_{y}^{0}\right)$ corresponds to the Schrödinger operator with an additional Dirichlet (Neumann) boundary condition at $y$. In obvious notation, $H_{y}^{\beta}$ decomposes into the direct sum of half-line operators

$$
H_{y}^{\beta}=H_{-, y}^{\beta} \oplus H_{+, y}^{\beta}
$$

with respect to

$$
L^{2}(\mathbb{R})=L^{2}((-\infty, y]) \oplus L^{2}([y, \infty))
$$

In particular, $H_{+, y}^{\beta}$ equals $H_{+, \alpha}$ for $\beta=\cot (\alpha)$ and $y=0$ in our notation (A.14), and, as indicated at the end of Appendix A, our (variable) reference point $x=y$ will be added as a subscript to obtain $\theta_{\alpha, y}(z, x), \phi_{\alpha, y}(z, x), \psi_{ \pm, \alpha, y}(z, x), m_{ \pm, \alpha, y}(z)$, $M_{\alpha, y}(z)$, etc. $H$ and $H_{y}^{\beta}$, defined in terms of separated boundary conditions, are real operators. Moreover, as observed in Appendix A, the point spectrum of $H$ is simple. 
Next, we recall a few results from [20]. With $G\left(z, x, x^{\prime}\right)$ and $G_{y}^{\beta}\left(z, x, x^{\prime}\right)$ the Green's functions of $H$ and $H_{y}^{\beta}$, one obtains

$$
\begin{gathered}
G_{y}^{\beta}\left(z, x, x^{\prime}\right)=G\left(z, x, x^{\prime}\right)-\frac{\left(\beta+\partial_{2}\right) G(z, x, y)\left(\beta+\partial_{1}\right) G\left(z, y, x^{\prime}\right)}{\left(\beta+\partial_{1}\right)\left(\beta+\partial_{2}\right) G(z, y, y)}, \\
\beta \in \mathbb{R}, z \in \mathbb{C} \backslash\left\{\sigma\left(H_{y}^{\beta}\right) \cup \sigma(H)\right\}, \\
G_{y}^{\infty}\left(z, x, x^{\prime}\right)=G\left(z, x, x^{\prime}\right)-G(z, y, y)^{-1} G(z, x, y) G\left(z, y, x^{\prime}\right), \\
z \in \mathbb{C} \backslash\left\{\sigma\left(H_{y}^{\infty}\right) \cup \sigma(H)\right\} .
\end{gathered}
$$

Here

$$
\begin{gathered}
\partial_{1} G\left(z, y, x^{\prime}\right):=\left.\partial_{x} G\left(z, x, x^{\prime}\right)\right|_{x=y}, \quad \partial_{2} G(z, x, y):=\left.\partial_{x^{\prime}} G\left(z, x, x^{\prime}\right)\right|_{x^{\prime}=y} \\
\partial_{1} \partial_{2} G(z, y, y):=\left.\partial_{x} \partial_{x^{\prime}} G\left(z, x, x^{\prime}\right)\right|_{x=y=x^{\prime}}, \quad \text { etc. }
\end{gathered}
$$

and

$$
\partial_{1} G(z, y, x)=\partial_{2} G(z, x, y), \quad x \neq y
$$

As a consequence,

$$
\begin{aligned}
& \operatorname{Tr}\left[\left(H_{y}^{\beta}-z\right)^{-1}-(H-z)^{-1}\right] \\
& \quad=-\frac{d}{d z} \ln \left[\left(\beta+\partial_{1}\right)\left(\beta+\partial_{2}\right) G(z, y, y)\right], \quad \beta \in \mathbb{R},
\end{aligned}
$$

$$
\operatorname{Tr}\left[\left(H_{y}^{\infty}-z\right)^{-1}-(H-z)^{-1}\right]=-\frac{d}{d z} \ln [G(z, y, y)] .
$$

In analogy to $G(z, y, y)$ (cf. (A.47)), also

$$
\left(\beta+\partial_{1}\right)\left(\beta+\partial_{2}\right) G(z, y, y) \text { is Herglotz }
$$

for each $y \in \mathbb{R}$. Hence, both admit exponential representations of the form

$$
\begin{gathered}
G(z, y, y)=\exp \left\{c_{\infty}(y)+\int_{\mathbb{R}}\left[\frac{1}{\lambda-z}-\frac{\lambda}{1+\lambda^{2}}\right] \xi^{\infty}(\lambda, y) d \lambda\right\}, \\
c_{\infty}(y) \in \mathbb{R}, \quad 0 \leq \xi^{\infty}(\lambda, y) \leq 1 \text { a.e., } \\
\xi^{\infty}(\lambda, y)=\lim _{\epsilon \downarrow 0} \pi^{-1} \operatorname{Im}\{\ln [G(\lambda+i \epsilon, y, y)]\} \quad \text { for a.e. } \lambda \in \mathbb{R}, \\
\left(\beta+\partial_{1}\right)\left(\beta+\partial_{2}\right) G(z, y, y) \\
=\exp \left\{c_{\beta}(y)+\int_{\mathbb{R}}\left[\frac{1}{\lambda-z}-\frac{\lambda}{1+\lambda^{2}}\right]\left[\xi^{\beta}(\lambda, y)+1\right] d \lambda\right\}, \quad \beta \in \mathbb{R},
\end{gathered}
$$




$$
\begin{gathered}
c_{\beta}(y) \in \mathbb{R}, \quad-1 \leq \xi^{\beta}(\lambda, y) \leq 0 \text { a.e., } \quad \beta \in \mathbb{R}, \\
\xi^{\beta}(\lambda, y)=\lim _{\epsilon \downarrow 0} \pi^{-1} \operatorname{Im}\left\{\ln \left[\left(\beta+\partial_{1}\right)\left(\beta+\partial_{2}\right) G(\lambda+i \epsilon, y, y)\right]\right\}-1, \quad \beta \in \mathbb{R},
\end{gathered}
$$

for each $y \in \mathbb{R}$. Moreover,

$$
\operatorname{Tr}\left[\left(H_{y}^{\beta}-z\right)^{-1}-(H-z)^{-1}\right]=-\int_{\mathbb{R}}(\lambda-z)^{-2} \xi^{\beta}(\lambda, y) d \lambda, \quad \beta \in \mathbb{R} \cup\{\infty\} .
$$

(Strictly speaking, the results (3.5)-(3.18) have been derived in [20] assuming $\tau$ to be in the l.p. case at $\pm \infty$. However, these results extend to our present setting without effort.)

For later purpose, we also note the identities (for each $y \in \mathbb{R}$ ),

$$
G(z, y, y)=M_{0, y, 2,2}(z)=\left[m_{-, 0, y}(z)-m_{+, 0, y}(z)\right]^{-1},
$$

$$
\begin{array}{r}
\sin ^{2}(\alpha)\left(\beta+\partial_{1}\right)\left(\beta+\partial_{2}\right) G(z, y, y)=M_{\alpha, y, 2,2}(z)=\left[m_{-, \alpha, y}(z)-m_{+, \alpha, y}(z)\right]^{-1}, \\
\beta=\cot (\alpha), \alpha \in(0, \pi),
\end{array}
$$

and especially

$$
\begin{aligned}
& m_{+, \alpha_{2}, y}(z)^{2}+\left\{\left[m_{-, \alpha_{2}, y}(z)-m_{+, \alpha_{2}, y}(z)\right]+2 \cot \left(\alpha_{1}-\alpha_{2}\right)\right\} m_{+, \alpha_{2}, y}(z) \\
& +\cot ^{2}\left(\alpha_{1}-\alpha_{2}\right)+\left[m_{-, \alpha_{2}, y}(z)-m_{+, \alpha_{2}, y}(z)\right] \cot \left(\alpha_{1}-\alpha_{2}\right) \\
& -\left[\sin \left(\alpha_{1}-\alpha_{2}\right)\right]^{-2}\left[m_{-, \alpha_{2}, y}(z)-m_{+, \alpha_{2}, y}(z)\right]\left[m_{-, \alpha_{1}, y}(z)-m_{+, \alpha_{1}, y}(z)\right]^{-1}=0, \\
& \alpha_{1} \neq \alpha_{2}, z \in \mathbb{C} \backslash \mathbb{R},
\end{aligned}
$$

following directly from (A.38).

As a consequence of Theorem 2.1, the basic uniqueness criterion for Schrödinger operators on $\mathbb{R}$ reads as follows.

Theorem 3.1. Suppose $\alpha_{1}, \alpha_{2} \in[0, \pi), \alpha_{1} \neq \alpha_{2}$, and assume $V_{j}, j=1,2$, satisfy hypothesis (3.1). Define $H_{j}, m_{ \pm, j, \alpha_{j}, y}(z), M_{j, \alpha_{j}, y}(z)$ associated with $\tau_{j}=-\frac{d^{2}}{d x^{2}}+$ $V_{j}(x), x \in \mathbb{R}, j=1,2$. Then the following are equivalent:

(i) $m_{+, 1, \alpha_{1}, y}(z)=m_{+, 2, \alpha_{2}, y}(z), m_{-, 1, \alpha_{1}, y}(z)=m_{-, 2, \alpha_{2}, y}(z), z \in \mathbb{C}_{+}$.

(ii) $M_{1, \alpha_{1}, y}(z)=M_{2, \alpha_{2}, y}(z), z \in \mathbb{C}_{+}$.

(iii) $\alpha_{1}=\alpha_{2}$ and $V_{1}(x)=V_{2}(x)$ for a.e. $x \in \mathbb{R}$.

The following is our principal characterization result for Schrödinger operators on $\mathbb{R}$.

Theorem 3.2. Let $\beta_{1}, \beta_{2} \in \mathbb{R} \cup\{\infty\}, \beta_{1} \neq \beta_{2}$, and $x_{0} \in \mathbb{R}$.

(i) $\xi^{\beta_{1}}\left(\lambda, x_{0}\right)$ and $\xi^{\beta_{2}}\left(\lambda, x_{0}\right)$ for a.e. $\lambda \in \mathbb{R}$ uniquely determine $V(x)$ for a.e. $x \in$ $\mathbb{R}$ if the pair $\left(\beta_{1}, \beta_{2}\right)$ differs from $(0, \infty),(\infty, 0)$.

(ii) If $\left(\beta_{1}, \beta_{2}\right)=(0, \infty)$ or $(\infty, 0)$, assume in addition that $\tau$ is in the limit point case at $+\infty$ and $-\infty$. Then $\xi^{\infty}\left(\lambda, x_{0}\right)$ and $\xi^{0}\left(\lambda, x_{0}\right)$ for a.e. $\lambda \in \mathbb{R}$ uniquely determine $V$ a.e. up to reflection symmetry with respect to $x_{0}$; that is, both $V(x), V\left(2 x_{0}-x\right)$ for a.e. $x \in \mathbb{R}$ correspond to $\xi^{\infty}\left(\lambda, x_{0}\right)$ and $\xi^{0}\left(\lambda, x_{0}\right)$ for a.e. $\lambda \in \mathbb{R}$. 
Proof. (i) Identifying $x_{0}$ and $y$ in (3.21), one can solve for $m_{+, \alpha_{2}, y}(z)$ to obtain

$$
\begin{aligned}
m_{+, \alpha_{2}, x_{0}}(z)= & -\frac{1}{2}\left[m_{-, \alpha_{2}, x_{0}}(z)-m_{+, \alpha_{2}, x_{0}}(z)\right]-\cot \left(\alpha_{1}-\alpha_{2}\right) \\
\pm & \left\{\frac{1}{4}\left[m_{-, \alpha_{2}, x_{0}}(z)-m_{+, \alpha_{2}, x_{0}}(z)\right]^{2}\right. \\
& \left.+\frac{1}{\sin ^{2}\left(\alpha_{1}-\alpha_{2}\right)} \frac{\left[m_{-, \alpha_{2}, x_{0}}(z)-m_{+, \alpha_{2}, x_{0}}(z)\right]}{\left[m_{-, \alpha_{1}, x_{0}}(z)-m_{+, \alpha_{1}, x_{0}}(z)\right]}\right\}^{1 / 2}, \quad z \in \mathbb{C} \backslash \mathbb{R} .
\end{aligned}
$$

By $(3.12),(3.15),(3.19)$, and $(3.20),\left[m_{-, \alpha_{j}, x_{0}}(z)-m_{+, \alpha_{j}, x_{0}}(z)\right]$ are both determined by $\xi^{\beta_{j}}\left(\lambda, x_{0}\right), \beta_{j}=\cot \left(\alpha_{j}\right), j=1,2$, respectively and hence the right-hand-side of (3.22) is determined up to the $+/-$ ambiguity. In order to resolve that ambiguity, we now consider the following case distinction:

a) $\alpha_{j} \in(0, \pi)$ (i.e., $\left.\beta_{j} \in \mathbb{R}\right), j=1,2$. Then by (A.39),

$$
m_{ \pm, \alpha_{2}, x_{0}}(z) \underset{z \rightarrow i \infty}{=} \cot \left(\alpha_{2}\right)+o\left(z^{-1 / 2}\right),
$$

which inserted into (3.22) results in

$m_{+, \alpha_{2}, x_{0}}(z) \underset{z \rightarrow i \infty}{=} \cot \left(\alpha_{2}-\alpha_{1}\right)+o\left(z^{-1 / 2}\right) \pm\left\{\frac{\sin ^{2}\left(\alpha_{1}\right)}{\sin ^{2}\left(\alpha_{1}-\alpha_{2}\right) \sin ^{2}\left(\alpha_{2}\right)}+O\left(z^{-1}\right)\right\}^{1 / 2}$.

A comparison of (3.23) and (3.24) reveals that only one choice of the sign (the + sign, choosing the branch of $\sqrt{\cdot}$ such that $\sqrt{x}>0$ for $x>0$ ) in (3.24) can be compatible with the leading behavior $\cot \left(\alpha_{2}\right)$ in (3.23). This resolves the sign ambiguity in (3.24) and hence in (3.22), and thus determines $m_{+, \alpha_{2}, x_{0}}(z)$. Since $\xi^{\beta_{2}}\left(\lambda, x_{0}\right)$ determines $\left[m_{-, \alpha_{2}, x_{0}}(z)-m_{+, \alpha_{2}, x_{0}}(z)\right], m_{-, \alpha_{2}, x_{0}}(z)$ is also determined. Thus, both Weyl $m$-functions $m_{ \pm, \alpha_{2}, x_{0}}(z)$ are known, and this in turn determines $V$ a.e. by Theorem 3.1 .

b) $\alpha_{2}=0$ (i.e., $\left.\beta_{2}=\infty\right), \alpha_{1} \neq \pi / 2$ (i.e., $\beta_{1} \neq 0$ ). Then by (A.40),

$$
m_{ \pm, 0, x_{0}}(z) \underset{z \rightarrow i \infty}{=} \pm i z^{1 / 2}+o(1)
$$

which inserted into (3.22) yields

$$
m_{+, 0, x_{0}}(z) \underset{z \rightarrow i \infty}{=} i z^{1 / 2}-\cot \left(\alpha_{1}\right)+o(1) \pm\{O(1)\}^{1 / 2} .
$$

Since by (3.25) the $\{O(1)\}^{1 / 2}$-term must cancel $-\cot \left(\alpha_{1}\right)$, this again resolves the sign ambiguity in (3.26) (once more the + sign turns out to be the right one) and hence in (3.22). Thus, $m_{+, 0, x_{0}}(z)$ is determined. Since $\xi^{\infty}\left(\lambda, x_{0}\right)$ determines $\left[m_{-, 0, x_{0}}(z)-m_{+, 0, x_{0}}(z)\right]$, also $m_{-, 0, x_{0}}(z)$ and hence $V$ is determined a.e. as in part a).

(ii) In the exceptional case where $\left(\beta_{1}, \beta_{2}\right)=(0, \infty),(\infty, 0)$, the exchange

$$
V(x) \rightarrow V\left(2 x_{0}-x\right) \text { implies } m_{ \pm, 0, x_{0}}(z) \rightarrow-m_{\mp, 0, x_{0}}(z),
$$


since we assumed the l.p. case at $\pm \infty$. This substitution leaves

$$
\left[m_{-, 0, x_{0}}(z)-m_{+, 0, x_{0}}(z)\right]^{-1}=G\left(z, x_{0}, x_{0}\right)
$$

and

$$
\begin{aligned}
& m_{-, 0, x_{0}}(z) m_{+, 0, x_{0}}(z)\left[m_{-, 0, x_{0}}(z)-m_{+, 0, x_{0}}(z)\right]^{-1} \\
& \quad=\left[m_{-, \pi / 2, x_{0}}(z)-m_{+, \pi / 2, x_{0}}(z)\right]^{-1}=\partial_{1} \partial_{2} G\left(z, x_{0}, x_{0}\right)
\end{aligned}
$$

and hence $\xi^{\infty}\left(\lambda, x_{0}\right)$ and $\xi^{0}\left(\lambda, x_{0}\right)$ invariant (cf. (3.19) and (3.20)). (Here we used that $m_{ \pm, \pi / 2, x_{0}}(z)=-\left[m_{ \pm, 0, x_{0}}(z)\right]^{-1}$, see (A.38).)

Corollary 3.3. Suppose $\tau$ is in the limit point case at $+\infty$ and $-\infty$, and let $\beta \in \mathbb{R} \cup\{\infty\}$ and $x_{0} \in \mathbb{R}$. Then $\xi^{\beta}\left(\lambda, x_{0}\right)$ for a.e. $\lambda \in \mathbb{R}$ uniquely determines $V(x)$ for a.e. $x \in \mathbb{R}$ if and only if $V$ is reflection symmetric with respect to $x_{0}$, that is, $V\left(2 x_{0}-x\right)=V(x)$ a.e.

Proof. First suppose that $V\left(2 x_{0}-x\right)=V(x)$ a.e. Then (A.38) yields

$$
m_{-, \alpha, x_{0}}(z)=-m_{+, \pi-\alpha, x_{0}}(z), \quad \alpha \in[0, \pi) .
$$

If $\beta \in \mathbb{R} \backslash\{0\}$ (i.e., $\alpha \in(0, \pi) \backslash\{\pi / 2\}, \beta=\cot (\alpha)$ ), then (3.30) implies

$$
\left[m_{-, \alpha, x_{0}}(z)-m_{+, \alpha, x_{0}}(z)\right]^{-1}=\left[m_{-, \pi-\alpha, x_{0}}(z)-m_{+, \pi-\alpha, x_{0}}(z)\right]^{-1} .
$$

By (3.15), this yields $\xi^{\beta}\left(\lambda, x_{0}\right)=\xi^{-\beta}\left(\lambda, x_{0}\right)$ a.e., and hence $V$ is uniquely determined a.e. by Theorem 3.2. On the other hand, if $\beta=\infty$ or 0 (i.e., $\alpha=0$ or $\pi / 2$ ), then (3.30) yields

$$
m_{-, 0, x_{0}}(z)=-m_{+, 0, x_{0}}(z) \quad \text { or } \quad m_{-, \pi / 2, x_{0}}(z)=-m_{+, \pi / 2, x_{0}}(z) .
$$

This determines $m_{ \pm, 0, x_{0}}(z)$ or $m_{ \pm, \pi / 2, x_{0}}(z)$ and hence $V$ a.e. by Theorem 3.1 .

Conversely, suppose $V$ is not reflection symmetric with respect to $x_{0}$. Define $\widehat{V}(x)=V\left(2 x_{0}-x\right)$ a.e. and denote by $\widehat{m}_{ \pm, \alpha, x_{0}}\left(z_{0}\right), \widehat{M}_{\alpha, x_{0}}(z)$, and $\widehat{\xi}^{\beta}\left(\lambda, x_{0}\right)$ the corresponding quantities associated with $\widehat{V}$. Then

$$
\widehat{m}_{ \pm, \pi-\alpha, x_{0}}(z)=-m_{\mp, \alpha, x_{0}}(z), \quad \alpha \in[0, \pi)
$$

(identifying $\alpha=0$ and $\pi$ ), and hence

$$
\widehat{M}_{\pi-\alpha, x_{0}}(z)=\left(\begin{array}{cc}
M_{\alpha, x_{0}, 1,1}(z) & -M_{\alpha, x_{0}, 1,2}(z) \\
-M_{\alpha, x_{0}, 2,1}(z) & M_{\alpha, x_{0}, 2,2}(z)
\end{array}\right) \neq M_{\alpha, x_{0}}(z)
$$

since $m_{-, \alpha, x_{0}}(z) \neq-m_{+, \alpha, x_{0}}(z)$ for all $\alpha \in[0, \pi)$. (The latter fact is obvious from the asymptotic behavior (A.39) for $\alpha \in(0, \pi) \backslash\{\pi / 2\}$, and also follows from our hypothesis that $V$ is not reflection symmetric w.r.t. $x_{0}$ for $\alpha=0, \pi / 2$. Alternatively, it also follows from our hypothesis and Theorem 3.1.) (3.34), however, shows that $\xi^{\beta}\left(\lambda, x_{0}\right)=\widehat{\xi}^{-\beta}\left(\lambda, x_{0}\right)$ is common to $V$ and $\widehat{V} \neq V$.

In view of Corollary 2.5, it seems appropriate to formulate Theorem 3.2 in the special case of purely discrete spectra. 
Corollary 3.4. Suppose $H$ (and hence $H_{y}^{\beta}$ for all $y \in \mathbb{R}, \beta \in \mathbb{R} \cup\{\infty\}$ ) has purely discrete spectrum, that is, $\sigma_{\mathrm{ess}}(H)=\emptyset$, and let $\beta_{1}, \beta_{2} \in \mathbb{R} \cup\{\infty\}, \beta_{1} \neq \beta_{2}$, and $x_{0} \in \mathbb{R}$.

(i) $\sigma(H), \sigma\left(H_{x_{0}}^{\beta_{j}}\right), j=1,2$, uniquely determine $V$ a.e. if the pair $\left(\beta_{1}, \beta_{2}\right)$ differs from $(0, \infty)$ and $(\infty, 0)$.

(ii) If $\left(\beta_{1}, \beta_{2}\right)=(0, \infty)$ or $(\infty, 0)$, assume in addition that $\tau$ is in the limit point case at $+\infty$ and $-\infty$. Then $\sigma(H), \sigma\left(H_{x_{0}}^{\infty}\right)$, and $\sigma\left(H_{x_{0}}^{0}\right)$ uniquely determine $V$ a.e. up to reflection symmetry with respect to $x_{0}$, that is, both $V(x)$ and $\widehat{V}(x)=V\left(2 x_{0}-x\right)$ for a.e. $x \in \mathbb{R}$ correspond to $\sigma(H)=\sigma(\widehat{H}), \sigma\left(H_{x_{0}}^{\infty}\right)=$ $\sigma\left(\widehat{H}_{x_{0}}^{\infty}\right)$, and $\sigma\left(H_{x_{0}}^{0}\right)=\sigma\left(\widehat{H}_{x_{0}}^{0}\right)$. Here, in obvious notation, $\widehat{H}, \widehat{H}_{x_{0}}^{\infty}, \widehat{H}_{x_{0}}^{0}$ correspond to $\widehat{\tau}=-\frac{d^{2}}{d x^{2}}+\widehat{V}(x), x \in \mathbb{R}$.

(iii) Suppose $\tau$ is in the limit point case at $+\infty$ and $-\infty$, and let $\beta \in \mathbb{R} \cup\{\infty\}$. Then $\sigma(H)$ and $\sigma\left(H_{x_{0}}^{\beta}\right)$ uniquely determine $V$ a.e. if and only if $V$ is reflection symmetric with respect to $x_{0}$.

(iv) Suppose that $V$ is reflection symmetric with respect to $x_{0}$ and $\tau$ is nonoscillatory at $+\infty$ and $-\infty$. Then $V$ is uniquely determined a.e. by $\sigma(H)$ in the sense that $V$ is the only potential symmetric with respect to $x_{0}$ with spectrum $\sigma(H)$.

Proof. (i) We denote $\sigma(H)=\left\{e_{n}\right\}_{n \in J_{0}}, \sigma\left(H_{x_{0}}^{\beta}\right)=\left\{\lambda_{n}^{\beta}\left(x_{0}\right)\right\}_{n \in I^{\beta}}$, where $I^{\beta}=J_{0}$, $\beta \in \mathbb{R}$, and $I^{\infty}=J$, with $J_{0}=\mathbb{N}_{0}$ or $\mathbb{Z}$ and $J=\mathbb{N}$ or $\mathbb{Z}$ depending on whether or not $H$ is bounded from below. Moreover, we use the ordering $e_{n}<e_{n+1}$, $\lambda_{n}^{\beta}\left(x_{0}\right) \leq \lambda_{n+1}^{\beta}\left(x_{0}\right)$. By general principles,

$$
\begin{gathered}
\lambda_{0}^{\beta}\left(x_{0}\right) \leq e_{0}, \quad \beta \in \mathbb{R} \text { if } H \text { is bounded from below, } \\
e_{n} \leq \lambda_{n}^{\beta}\left(x_{0}\right) \leq e_{n+1}, \quad \beta \in \mathbb{R} \cup\{\infty\} .
\end{gathered}
$$

By hypothesis, $\xi^{\beta}\left(\lambda, x_{0}\right), \beta \in \mathbb{R} \cup\{\infty\}$, is a pure step function which jumps by +1 at every (necessarily simple) eigenvalue of $H$ (since $\psi_{+, \alpha, x_{0}}\left(e_{m}, x\right)$ and $\psi_{-, \tilde{\alpha}, x_{0}}\left(e_{m}, x\right)$ for $e_{m} \in \sigma(H), \alpha, \tilde{\alpha} \in[0, \pi)$, are unique up to constant multiples). Similarly, $\xi^{\beta}\left(\lambda, x_{0}\right)$ jumps by $-m\left(\lambda_{n}^{\beta}\left(x_{0}\right)\right)(m(\lambda)$ denotes the multiplicity of an eigenvalue $\lambda)$ at any eigenvalue of $H_{x_{0}}^{\beta}$. As long as all multiplicities involved are equal to one, that is,

$$
m\left(\lambda_{n}^{\beta_{j}}\left(x_{0}\right)\right)=1, \quad n \in I^{\beta_{j}},
$$

$\sigma(H), \sigma\left(H_{x_{0}}^{\beta_{1}}\right)$, and $\sigma\left(H_{x_{0}}^{\beta_{2}}\right)$ clearly determine $\xi^{\beta_{j}}\left(\lambda, x_{0}\right), j=1,2$. The case where some eigenvalues of $H_{x_{0}}^{\beta_{j}}$ are degenerate needs a bit more care. Assume, for example,

$$
\lambda_{m_{0}}^{\beta_{1}}\left(x_{0}\right)=\lambda_{m_{0}+1}^{\beta_{1}}\left(x_{0}\right):=e_{m_{0}}, \quad \text { i.e., } m\left(e_{m_{0}}\right)=2
$$

for some $m_{0} \in I^{\beta_{1}}$. Since half-line spectra are necessarily simple, (3.37) implies that $H_{+, x_{0}}^{\beta_{1}}$ and $H_{-, x_{0}}^{\beta_{1}}$, the corresponding half-line operators in $L^{2}\left(\left(x_{0}, \pm \infty\right)\right)$ (cf. (3.3), (3.4)) associated with $H_{x_{0}}^{\beta_{1}}$, have the same simple eigenvalue $e_{m_{0}}$. As a consequence, $H$ itself has $e_{m_{0}}$ as a (simple) eigenvalue, that is, $e_{m_{0}} \in \sigma(H)$. Thus, $\xi^{\beta_{1}}\left(\lambda, x_{0}\right)$ jumps by $-2+1=-1$ at $\lambda_{m_{0}}^{\beta_{1}}\left(x_{0}\right)$ and stays -1 until $e_{m_{0}+1} \in \sigma(H)$.

Similarly, suppose $\lambda_{m_{0}}^{\beta_{1}}\left(x_{0}\right)=e_{m_{0}-1}$ for some $m_{0} \in I^{\beta_{1}}$ and let $\psi_{+, \alpha_{1}, x_{0}}\left(e_{m_{0}}, x\right)$ $=$ const. $\psi_{-, \alpha_{1}, x_{0}}\left(e_{m_{0}-1}, x\right), \beta_{1}=\cot \left(\alpha_{1}\right)$, be the unique eigenfunction of $H$ associated with $e_{m_{0}-1}$. Then also $\lambda_{m_{0}-1}^{\beta_{1}}\left(x_{0}\right)=e_{m_{0}-1}$, since the restrictions of 
$\psi_{ \pm, \alpha_{1}, x_{0}}\left(e_{m_{0}-1}, x\right)$ to $x \leq x_{0}$ and $x \geq x_{0}$ are eigenfunctions of $H_{-, x_{0}}^{\beta_{1}}$ and $H_{+, x_{0}}^{\beta_{1}}$, respectively. Hence $\sigma(H), \sigma\left(H_{x_{0}}^{\beta_{1}}\right)$, and $\sigma\left(H_{x_{0}}^{\beta_{2}}\right)$ determine $\xi^{\beta_{j}}\left(\lambda, x_{0}\right), j=1,2$, and we may apply Theorem 3.2(i).

(ii) now follows from Theorem 3.2(ii), and (iii) is clear from Corollary 3.3. (iv) is a consequence of (iii), the fact that $\tau$ being non-oscillatory at $\pm \infty$ implies the l.p. case at $\pm \infty$, and the ordering

$$
\begin{gathered}
\lambda_{0}^{0}\left(x_{0}\right)=e_{0}, \quad \lambda_{2 m+1}^{\infty}\left(x_{0}\right)=e_{2 m+1}=\lambda_{2 m+2}^{\infty}\left(x_{0}\right), \\
\lambda_{2 m+1}^{0}\left(x_{0}\right)=e_{2 m+2}=\lambda_{2 m+2}^{0}\left(x_{0}\right), \quad m \in \mathbb{N}_{0} .
\end{gathered}
$$

We emphasize that Corollary 3.4(iii) is, of course, implied by the result of Borg [5] and Marchenko [32] (see Corollary 2.5 with $\alpha_{1}=0, \alpha_{2}=\pi / 2$ ).

So far, we have exclusively dealt with $\xi$-functions and spectra in connection with uniqueness theorems. A variety of further uniqueness results can be obtained by invoking alternative information such as the left/right distribution of $\lambda_{n}^{\beta}\left(x_{0}\right)$ (i.e., whether $\lambda_{n}^{\beta}\left(x_{0}\right)$ is an eigenvalue of $H_{-, x_{0}}^{\beta}$ in $L^{2}\left(\left(-\infty, x_{0}\right]\right)$ or of $H_{+, x_{0}}^{\beta}$ in $\left.L^{2}\left(\left[x_{0}, \infty\right)\right)\right)$ and/or associated norming constants. For brevity we concentrate on only one such case, the Dirichlet boundary condition $\beta=\infty$.

We start by introducing Dirichlet data instead of merely Dirichlet eigenvalues. For notational convenience we now denote the Dirichlet eigenvalues $\lambda_{n}^{\infty}\left(x_{0}\right)$ by

$$
\mu_{n}\left(x_{0}\right), \quad n \in J,
$$

with $J \subseteq \mathbb{N}$ or $\mathbb{Z}$ an appropriate index set. Let $(a, b) \subseteq \mathbb{R} \backslash \sigma(H)$ be a spectral gap of $H$ and assume $\mu_{n}\left(x_{0}\right) \in(a, b)$. The corresponding Dirichlet datum is then defined by

$$
\left(\mu_{n}\left(x_{0}\right), \sigma_{n}\left(x_{0}\right)\right), \quad \sigma_{n}\left(x_{0}\right) \in\{-,+\},
$$

where $\sigma_{n}\left(x_{0}\right)=-/+$ records whether $\mu_{n}\left(x_{0}\right)$ is a left/right Dirichlet eigenvalue (i.e., an eigenvalue of $H_{-, x_{0}}^{\infty}$, respectively $H_{+, x_{0}}^{\infty}$ ).

A combination of $\xi$-functions and Dirichlet data allows one to rephrase the celebrated uniqueness theorem of Borg [4] for periodic potentials as follows. Assume in addition to hypothesis (3.1) that $V$ is periodic with period $\Omega>0$. Then Floquet theory yields that the spectra of $H$ and $H_{x_{0}}^{\infty}$ are of the type

$$
\begin{aligned}
\sigma(H) & =\bigcup_{n \in \mathbb{N}}\left[E_{2(n-1)}, E_{2 n-1}\right], \quad E_{0}<E_{1} \leq E_{2}<E_{3} \leq \cdots, \\
\sigma\left(H_{x_{0}}^{\infty}\right) & =\sigma(H) \cup\left\{\mu_{n}\left(x_{0}\right)\right\}_{n \in \mathbb{N}}, \quad E_{2 n-1} \leq \mu_{n}\left(x_{0}\right) \leq E_{2 n}, n \in \mathbb{N} .
\end{aligned}
$$

Let $I\left(x_{0}\right) \subseteq \mathbb{N}$ denote the set of all indices $j$ such that

$$
\left.\mu_{j}\left(x_{0}\right) \notin\left\{E_{n}\right\}_{n \in \mathbb{N}_{0}} \quad \text { (i.e., } \mu_{j}\left(x_{0}\right) \notin \sigma(H)\right) .
$$

Then Borg's result can be rephrased as follows. 
Theorem 3.5 (Borg [4], see also [34],[35]). Let $V \in L_{\text {loc }}^{1}(\mathbb{R})$ be real-valued and periodic of period $\Omega>0$. Then $\xi^{\infty}\left(\lambda, x_{0}\right)$ for a.e. $\lambda \in \mathbb{R}$ and $\sigma_{j}\left(x_{0}\right), j \in I\left(x_{0}\right)$, uniquely determine $V$ for a.e. $x \in \mathbb{R}$.

For the proof, it suffices to note that (cf., e.g., [15],[20],[26])

$$
\xi^{\infty}\left(\lambda, x_{0}\right)= \begin{cases}\frac{1}{2}, & \lambda \in\left(E_{2(n-1)}, E_{2 n-1}\right), n \in \mathbb{N} \\ 1, & \lambda \in\left(E_{2 n-1}, \mu_{n}\left(x_{0}\right)\right), n \in \mathbb{N} \\ 0, & \lambda \in\left(-\infty, E_{0}\right),\left(\mu_{n}\left(x_{0}\right), E_{2 n}\right), n \in \mathbb{N}\end{cases}
$$

in connection with the periodic case (3.41), (3.42). This result extends to algebrogeometric quasi-periodic finite-gap potentials and certain classes of almost-periodic potentials; we omit further details at this point.

After this warm-up we turn to a new uniqueness result for operators with purely discrete spectra. Assume

$$
\sigma_{\text {ess }}(H)=\emptyset \text { and denote } \sigma(H)=\left\{e_{n}\right\}_{n \in J_{0}}
$$

such that

$$
\sigma\left(H_{x_{0}}^{\infty}\right)=\left\{\mu_{n}\left(x_{0}\right)\right\}_{n \in J}, \quad e_{n-1} \leq \mu_{n}\left(x_{0}\right) \leq e_{n}, n \in J
$$

where $J_{0}=\mathbb{N}_{0}$ or $\mathbb{Z}$ and $J=\mathbb{N}$ or $\mathbb{Z}$ are appropriate index sets depending on whether or not $H$ is bounded from below.

Next we divide the spectrum of $H_{x_{0}}^{\infty}$ into simple and (twice) degenerate Dirichlet eigenvalues, that is, those which are disjoint from $\sigma(H)$ and those which coincide with an element of $\sigma(H)$,

$$
\begin{gathered}
J=I\left(x_{0}\right) \cup I^{\prime}\left(x_{0}\right), \quad I\left(x_{0}\right) \cap I^{\prime}\left(x_{0}\right)=\emptyset, \\
\left\{\mu_{j}\left(x_{0}\right)\right\}_{j \in I\left(x_{0}\right)} \cap \sigma(H)=\emptyset, \quad\left\{\mu_{j^{\prime}}\left(x_{0}\right)\right\}_{j^{\prime} \in I^{\prime}\left(x_{0}\right)} \subset \sigma(H)
\end{gathered}
$$

(i.e., $\mu_{j^{\prime}}\left(x_{0}\right) \in\left\{e_{j^{\prime}-1}, e_{j^{\prime}}\right\}$ for $\left.j^{\prime} \in I^{\prime}\left(x_{0}\right)\right)$. As a last ingredient we need the norming constants associated with the (twice) degenerate Dirichlet eigenvalues $\left\{\mu_{j^{\prime}}\left(x_{0}\right)\right\}_{j^{\prime} \in I^{\prime}\left(x_{0}\right)}$ denoted by

$$
c_{ \pm, j^{\prime}}\left(x_{0}\right)>0, \quad j^{\prime} \in I^{\prime}\left(x_{0}\right)
$$

Quite generally, the norming constant $c_{+, n}\left(x_{0}\right)>0$ (respectively $c_{-, n}\left(x_{0}\right)>0$ ) associated with $\mu_{n}\left(x_{0}\right) \in \sigma\left(H_{+, x_{0}}^{\infty}\right)$ (respectively $\mu_{n}\left(x_{0}\right) \in \sigma\left(H_{-, x_{0}}^{\infty}\right)$ ) is given by minus (respectively plus) the residue of the corresponding Weyl $m$-function $m_{+, 0, x_{0}}(z)$ (respectively $m_{-, 0, x_{0}}(z)$ ) at $z=\mu_{n}\left(x_{0}\right)$. Equivalently, one has

$$
c_{ \pm, n}\left(x_{0}\right)=\left\|\phi_{0, x_{0}}\left(\mu_{n}\left(x_{0}\right), \cdot\right)\right\|_{L^{2}\left(\mathbb{R}_{ \pm}\right)}^{-2}
$$

(cf. (A.37)).

Given these preparations we can state the following result. 
Theorem 3.6. Let $x_{0} \in \mathbb{R}$ and suppose $H$ has purely discrete spectrum, that is, $\sigma_{\text {ess }}(H)=\emptyset, \sigma(H)=\left\{e_{n}\right\}_{n \in J_{0}}$. Then $\xi^{\infty}\left(\lambda, x_{0}\right)$ for a.e. $\lambda \in \mathbb{R}, \sigma_{j}\left(x_{0}\right), j \in I\left(x_{0}\right)$, and $c_{+, j^{\prime}}\left(x_{0}\right), c_{-, j^{\prime}}\left(x_{0}\right), j^{\prime} \in I^{\prime}\left(x_{0}\right)$, uniquely determine $V$ for a.e. $x \in \mathbb{R}$.

Proof. The step function $\xi^{\infty}\left(\lambda, x_{0}\right)$ determines the Green's function $G\left(z, x_{0}, x_{0}\right)$ of $H$ by (3.12), and hence

$$
\left[m_{-, 0, x_{0}}(z)-m_{+, 0, x_{0}}(z)\right]=G\left(z, x_{0}, x_{0}\right)^{-1}
$$

is determined. Since $\sigma_{\text {ess }}(H)=\emptyset$, both $m_{ \pm, 0, x_{0}}(z)$ are meromorphic (on $\mathbb{C}$ ) with first-order poles (and zeros) on $\mathbb{R}$. Since by hypothesis we know the left/right distribution of all simple Dirichlet eigenvalues $\left\{\mu_{j}\left(x_{0}\right)\right\}_{j \in I\left(x_{0}\right)}$, we can infer the corresponding residue of $m_{-, 0, x_{0}}(z)$ (respectively $m_{+, 0, x_{0}}(z)$ ) from the knowledge of $G\left(z, x_{0}, x_{0}\right)^{-1}=\left[m_{-, 0, x_{0}}(z)-m_{+, 0, x_{0}}(z)\right]$. But for the remaining (twice) degenerate Dirichlet eigenvalues $\left\{\mu_{j^{\prime}}\left(x_{0}\right)\right\}_{j^{\prime} \in I^{\prime}\left(x_{0}\right)}$ of $H_{x_{0}}^{\infty}$, the residue of $m_{ \pm,-, x_{0}}(z)$ at $z=\mu_{j^{\prime}}\left(x_{0}\right), j^{\prime} \in I^{\prime}\left(x_{0}\right)$, equals $\mp c_{ \pm, j^{\prime}}\left(x_{0}\right)$ and hence is known as well. Thus, the principal parts of $m_{ \pm, 0, x_{0}}(z)$ are determined. Since the corresponding half-line spectral measures $d \rho_{ \pm, 0, x_{0}}(\lambda)$ associated with $H_{ \pm, x_{0}}^{\infty}=H_{ \pm, 0, x_{0}}$ are pure point measures supported on $\sigma\left(H_{ \pm, 0, x_{0}}\right)$ of corresponding mass $c_{ \pm, n}\left(x_{0}\right)$, they are completely determined under our hypothesis. But $d \rho_{ \pm, 0, x_{0}}(\lambda)$ uniquely determines $V$ a.e. on $\left[x_{0}, \pm \infty\right)$ by Theorem 2.1 .

If in addition $V$ is symmetric with respect to $x_{0}$ and $\tau$ is in the limit point case at $+\infty$ and $-\infty$, then $I\left(x_{0}\right)=\emptyset, I^{\prime}\left(x_{0}\right)=J, m_{+, 0, x_{0}}(z)=-m_{-, 0, x_{0}}(z)$, and hence $\xi^{\infty}\left(\lambda, x_{0}\right)$ alone uniquely determines $V$ a.e., recovering again the result of Borg [5] and Marchenko [32] recorded in Corollary 3.4(iii).

The reader might want to compare our method of proof of Theorem 3.6 with the inverse spectral approach to confining potentials on the half-line $\mathbb{R}_{+}$as presented in $[21]$.

\section{ACKNOWLEDGMENT}

F.G. is indebted to the Department of Mathematics at Caltech for its hospitality and support during the summers of 1993 and 1994 where some of this work was done.

\section{Appendix A. Herglotz Functions And Weyl-Titchmarsh theory}

We briefly summarize a few basic facts on Herglotz functions and then recall some of the essential elements of the Weyl-Titchmarsh theory for Schrödinger operators on the half-line $[0, \infty)$ as well as on $\mathbb{R}$ relevant in Sections 2 and 3.

We start with Herglotz functions (also called Pick or Nevanlinna-Pick functions). Denoting $\mathbb{C}_{ \pm}:=\{z \in \mathbb{C} \mid \pm \operatorname{Im}(z)>0\}$, any analytic map $m: \mathbb{C}_{+} \rightarrow \mathbb{C}_{+}$is called Herglotz. One conveniently defines $m$ on $\mathbb{C}_{-}$by $m(\bar{z})=\overline{m(z)}$ for $z \in \mathbb{C}_{+}$. Herglotz functions admit particular representations (Borel transforms) in terms of certain measures on $\mathbb{R}$. Since this aspect is of fundamental importance in the context of inverse spectral theory of Schrödinger operators, we recall the following classical results of Aronszajn and Donoghue [2]. 
Theorem A.1 [2]. Let $m$ be a Herglotz function. Then,

(i) There exist a measure $d \rho$ on $\mathbb{R}$ and a real-valued $\xi \in L_{\text {loc }}^{1}(\mathbb{R})$ such that

$$
\begin{aligned}
m(z) & =a+b z+\int_{\mathbb{R}}\left[\frac{1}{\lambda-z}-\frac{\lambda}{1+\lambda^{2}}\right] d \rho(\lambda) \\
& =\exp \left\{c+\int_{R}\left[\frac{1}{\lambda-z}-\frac{\lambda}{1+\lambda^{2}}\right] \xi(\lambda) d \lambda\right\},
\end{aligned}
$$

where

$$
\int_{\mathbb{R}} \frac{d \rho(\lambda)}{1+\lambda^{2}}<\infty, \quad a=\operatorname{Re}[m(i)], b \geq 0
$$

and

$$
0 \leq \xi \leq 1 \text { a.e., } \quad c=\operatorname{Re}\{\ln [m(i)]\}
$$

(ii) (Fatou's lemma)

$$
\begin{gathered}
\rho((\lambda, \mu])=\lim _{\delta \downarrow 0} \lim _{\epsilon \downarrow 0} \pi^{-1} \int_{\lambda+\delta}^{\mu+\delta} d \nu \operatorname{Im}[m(\nu+i \epsilon)], \\
\xi(\lambda)=\lim _{\epsilon \downarrow 0} \pi^{-1} \operatorname{Im}\{\ln [m(\lambda+i \epsilon)]\} \text { a.e. }
\end{gathered}
$$

(iii) Let $m, n \in \mathbb{N}$ and $b=0$. Then

$$
\int_{-\infty}^{0}\left(1+\lambda^{2}\right)^{-1}|\lambda|^{m}|\xi(\lambda)| d \lambda+\int_{0}^{\infty}\left(1+\lambda^{2}\right)^{-1}|\lambda|^{n}|\xi(\lambda)| d \lambda<\infty
$$

if and only if

$$
\begin{gathered}
\int_{-\infty}^{0}\left(1+\lambda^{2}\right)^{-1}|\lambda|^{m} d \rho(\lambda)+\int_{0}^{\infty}\left(1+\lambda^{2}\right)^{-1}|\lambda|^{n} d \rho(\lambda)<\infty \\
\text { and } \lim _{z \rightarrow i \infty} m(z)=a-\int_{\mathbb{R}}\left(1+\lambda^{2}\right)^{-1} \lambda d \rho(\lambda)>0 .
\end{gathered}
$$

(iv)

$$
m(z)=1+\int_{\mathbb{R}}(\lambda-z)^{-1} d \rho(\lambda) \quad \text { with } \int_{\mathbb{R}} d \rho(\lambda)<\infty
$$

if and only if

(A.10) $m(z)=\exp \left[\int_{\mathbb{R}}(\lambda-z)^{-1} \xi(\lambda) d \lambda\right] \quad$ with $0 \leq \xi \leq 1$ a.e. and $\xi \in L^{1}(\mathbb{R})$. 
In this case

$$
\int_{\mathbb{R}} d \rho(\lambda)=\int_{\mathbb{R}} \xi(\lambda) d \lambda
$$

(v) Any poles and zeros of $m$ are simple and located on the real axis, the residues at poles being negative.

The link between Herglotz functions and rank-one perturbations of self-adjoint operators is developed in detail in [38]. In particular, its universal applicability and unifying aspects in connection with the spectral theory of ordinary differential operators and finite-difference operators are amply illustrated in [16], [25],[38].

Next we turn to Schrödinger operators on the half-line $\mathbb{R}_{+}:=[0, \infty)$. The following material can be found, for example, in [6],[31], and [36]. Suppose

$$
V \in L^{1}([0, R]) \text { for all } R>0, \quad V \text { real-valued }
$$

and introduce the differential expression

$$
\tau_{+}=-\frac{d^{2}}{d x^{2}}+V(x), \quad x \geq 0 .
$$

Associated with $\tau_{+}$we introduce the following self-adjoint operator $H_{+, \alpha}$ in $L^{2}\left(\mathbb{R}_{+}\right)$. Pick a $z_{+} \in \mathbb{C} \backslash \mathbb{R}$ and a solution $f_{+}\left(z_{+}, \cdot\right) \in L^{2}\left(\mathbb{R}_{+}\right)$of $\tau_{+} \psi=z_{+} \psi$ (the existence of such an $f_{+}\left(z_{+}, x\right)$ is a fundamental result of Weyl's theory), and define

$$
\begin{gathered}
H_{+, \alpha} f=\tau_{+} f, \quad \alpha \in[0, \pi) \\
f \in \mathcal{D}\left(H_{+, \alpha}\right)=\left\{g \in L^{2}\left(\mathbb{R}_{+}\right) \mid g, g^{\prime} \in A C([0, R]) \text { for all } R>0\right. \\
\left.\sin (\alpha) g^{\prime}\left(0_{+}\right)+\cos (\alpha) g\left(0_{+}\right)=0 ; \lim _{R \rightarrow \infty} W\left(f_{+}\left(z_{+}\right), g\right)(R)=0 ; \tau_{+} g \in L^{2}\left(\mathbb{R}_{+}\right)\right\} .
\end{gathered}
$$

Here $W(f, g)(x)=f(x) g^{\prime}(x)-f^{\prime}(x) g(x)$ denotes the Wronskian of $f$ and $g$ and the boundary condition $\lim _{R \rightarrow \infty} W\left(f_{+}\left(z_{+}\right), g\right)=0$ at $x=+\infty$ can be omitted if and only if $\tau_{+}$is in the limit point (l.p.) case at $+\infty$, that is, if and only if $f_{+}\left(z_{+}, x\right)$ is unique (up to constant multiples). If $\tau_{+}$is in the limit circle (l.c.) case at $+\infty, H_{+, \alpha}$ depends on the choice of $f_{+}\left(z_{+}, x\right)$ and for definiteness we shall "fix the boundary condition at $+\infty, "$ that is, always employ the same $f_{+}\left(z_{+}, \cdot\right)$ in the definition (A.14) of $H_{+, \alpha}$ for all values of $\alpha \in[0, \pi)$. Due to our choice of (symmetric) separated boundary conditions in (A.14), $H_{+, \alpha}$ is a real operator (i.e., $g \in \mathcal{D}\left(H_{+, \alpha}\right)$ implies $\bar{g} \in \mathcal{D}\left(H_{+, \alpha}\right)$ and $\left.H_{+, \alpha} \bar{g}=\overline{\left(H_{+, \alpha} g\right)}\right)$, see, for example, [36], Section 6.4, with uniform spectral multiplicity one, cf. [10], Corollary XIII.5.5.

Next we introduce the fundamental system $\phi_{\alpha}(z, x), \theta_{\alpha}(z, x), z \in \mathbb{C}$, of solutions of

$$
\tau_{+} \psi(z, x)=z \psi(z, x), \quad x \geq 0
$$

satisfying

$$
\phi_{\alpha}(z, 0)=-\theta_{\alpha}^{\prime}(z, 0)=-\sin (\alpha), \quad \phi_{\alpha}^{\prime}(x, 0)=\theta_{\alpha}(z, 0)=\cos (\alpha)
$$


such that $W\left(\theta_{\alpha}(z), \phi_{\alpha}(z)\right)=1$. Furthermore, let $\psi_{+, \alpha}(z, x), z \in \mathbb{C} \backslash \mathbb{R}$, be the unique solution of (A.15) which satisfies

$$
\begin{gathered}
\psi_{+, \alpha}(z, \cdot) \in L^{2}\left(\mathbb{R}_{+}\right), \quad \sin (\alpha) \psi_{+, \alpha}^{\prime}\left(z, 0_{+}\right)+\cos (\alpha) \psi_{+, \alpha}\left(z, 0_{+}\right)=1, \\
\lim _{R \rightarrow \infty} W\left(f_{+}\left(z_{+}\right), \psi_{+, \alpha}(z)\right)(R)=0, \quad z \in \mathbb{C} \backslash \mathbb{R}
\end{gathered}
$$

(the latter condition being superfluous, i.e., automatically fulfilled, if $\tau_{+}$is l.p. at $+\infty)$. Uniqueness of $\psi_{+, \alpha}(z, x)$ is a consequence of Weyl's theory and the fact that we are imposing conditions separately at 0 and $\infty$ in (A.17); see, for example, [10], Theorem XIII.2.32. $\psi_{+, \alpha}(z, x)$ is of the form

$$
\psi_{+, \alpha}(z, x)=\theta_{\alpha}(z, x)+m_{+, \alpha}(z) \phi_{\alpha}(z, x)
$$

with $m_{+, \alpha}(z)$ being Weyl's $m$-function. $m_{+, \alpha}(z)$ is well known to be a Herglotz function (cf. also the comment following (A.27)). To avoid repetitions, we list properties of $m_{+, \alpha}(z)$ a bit later (together with those of $m_{-, \alpha}(z)$ ). Here we just note that the Herglotz property of $m_{+, \alpha}(z)$ together with the asymptotic behavior (A.39), (A.40) yields the existence of a measure $d \rho_{+, \alpha}$ such that

$$
\begin{aligned}
m_{+, \alpha} & =a_{+, \alpha}+\int_{\mathbb{R}}\left[\frac{1}{\lambda-z}-\frac{\lambda}{1+\lambda^{2}}\right] d \rho_{+, \alpha}(\lambda), & & \alpha \in[0, \pi), \\
& =\cot (\alpha)+\int_{\mathbb{R}}(\lambda-z)^{-1} d \rho_{+, \alpha}(\lambda), & & \alpha \in(0, \pi),
\end{aligned}
$$

with

$$
\int_{\mathbb{R}} \frac{d \rho_{+, \alpha}(\lambda)}{1+|\lambda|} \begin{cases}<\infty, & \alpha \in(0, \pi), \\ =\infty, & \alpha=0 .\end{cases}
$$

The Green's function $G_{+, \alpha}\left(z, x, x^{\prime}\right)$ of $H_{+, \alpha}$ finally reads

$$
\begin{aligned}
& \left(\left(H_{+, \alpha}-z\right)^{-1} f\right)(x)=\int_{0}^{\infty} d x^{\prime} G_{+, \alpha}\left(z, x, x^{\prime}\right) f\left(x^{\prime}\right), \\
& z \in \mathbb{C} \backslash \sigma\left(H_{+, \alpha}\right), f \in L^{2}\left(\mathbb{R}_{+}\right), \\
& G_{+, \alpha}\left(z, x, x^{\prime}\right)= \begin{cases}\phi_{\alpha}(z, x) \psi_{+, \alpha}\left(z, x^{\prime}\right), & 0 \leq x \leq x^{\prime}, \\
\phi_{\alpha}\left(z, x^{\prime}\right) \psi_{+, \alpha}(z, x), & 0 \leq x^{\prime} \leq x,\end{cases} \\
& =\int_{\mathbb{R}}(\lambda-z)^{-1} \phi_{\alpha}(\lambda, x) \phi_{\alpha}\left(\lambda, x^{\prime}\right) d \rho_{+, \alpha}(\lambda),
\end{aligned}
$$

where $\sigma(\cdot)$ denotes the spectrum. In particular, (A.18), (A.23), and (A.24) yield

$$
\begin{aligned}
G_{+, \alpha}(z, 0,0) & =-\sin (\alpha)\left[\cos (\alpha)-m_{+, \alpha}(z) \sin (\alpha)\right], & & \alpha \in[0, \pi), \\
& =\sin ^{2}(\alpha) \int_{\mathbb{R}}(\lambda-z)^{-1} d \rho_{+, \alpha}(\lambda), & & \alpha \in(0, \pi),
\end{aligned}
$$


and for each $x \geq 0$,

$$
G_{+, \alpha}(z, x, x) \text { is Herglotz. }
$$

While the latter result is obvious from (A.24) (note we have $\phi_{\alpha}(\lambda, x) \underset{|\lambda| \rightarrow \infty}{=} O(1)$ for $\alpha \in(0, \pi)$ and $\phi_{0}(\lambda, x) \underset{|\lambda| \rightarrow \infty}{=} O\left(|\lambda|^{-1 / 2}\right)$ for fixed $\left.x \in \mathbb{R}\right)$, the fact (A.27) is easily proved directly using the first resolvent equation and self-adjointness of $H_{+, \alpha}$. (This statement holds quite generally for the diagonal integral kernel of resolvents of selfadjoint operators in connection with general measure spaces as long as the diagonal kernel is well-defined. In particular, it holds for the diagonal Green's function of finite difference operators.) Together with (A.25) this yields a direct proof that $m_{+, \alpha}(z)$ is Herglotz too.

Finally, we recall a few facts in connection with Schrödinger operators on $\mathbb{R}$. Assuming

$$
V \in L_{\mathrm{loc}}^{1}(\mathbb{R}), \quad V \text { real-valued, }
$$

one introduces the differential expression

$$
\tau=-\frac{d^{2}}{d x^{2}}+V(x), \quad x \in \mathbb{R},
$$

and picks $z_{ \pm} \in \mathbb{C} \backslash \mathbb{R}$ and solutions $f_{ \pm}\left(z_{ \pm}, \cdot\right) \in L^{2}\left(\mathbb{R}_{ \pm}\right)\left(\mathbb{R}_{-}:=(-\infty, 0]\right)$ of $\tau \psi(z)=$ $z \psi(z)$ for $z=z_{+}$, respectively $z_{-}$. One then defines a self-adjoint operator $H$ in $L^{2}(\mathbb{R})$ by

$$
\begin{aligned}
& H f=\tau f, \\
& f \in \mathcal{D}(H)=\left\{g \in L^{2}(\mathbb{R}) \mid g, g^{\prime} \in A C_{\mathrm{loc}}(\mathbb{R}) ;\right. \\
& \left.\qquad \lim _{R \rightarrow \pm \infty} W\left(f_{ \pm}\left(z_{ \pm}\right), g\right)(R)=0 ; \tau g \in L^{2}(\mathbb{R})\right\},
\end{aligned}
$$

where again, the boundary condition at $+\infty$ (or $-\infty)$ can be omitted if and only if $\tau$ is l.p. at $+\infty$ (or $-\infty)$, that is, if and only if $f_{+}\left(z_{+}, \cdot\right)$ (or $f_{-}\left(z_{-}, \cdot\right)$ ) is unique up to constant multiples. Again, when considering restrictions of $\tau$ to $\mathbb{R}_{ \pm}$, we shall fix the boundary condition at $+\infty$ and/or $-\infty$ if $\tau$ is l.c. at $+\infty$ and/or $-\infty$. As in the half-line case (A.14), the separated boundary conditions in (A.30) imply that $H$ is a real operator (see, e.g., [36], Section 6.4). Moreover, the point spectrum $\sigma_{p}(H)$ of $H$ (the set of eigenvalues of $H$ ) is simple (this follows, e.g., from [10], Theorem XIII.2.32).

Next we define $\phi_{\alpha}(z, x), \theta_{\alpha}(z, x)$ as in (A.15), (A.16) (replacing $\tau_{+}$by $\tau$ ) and introduce the uniquely determined solutions $\psi_{ \pm, \alpha}(z, x)$ of

$$
\tau \psi(z, x)=z \psi(z, x), \quad x \in \mathbb{R},
$$

satisfying

$$
\begin{gathered}
\psi_{ \pm, \alpha}(z, \cdot) \in L^{2}\left(\mathbb{R}_{ \pm}\right), \quad \sin (\alpha) \psi_{ \pm, \alpha}^{\prime}(z, 0)+\cos (\alpha) \psi_{ \pm, \alpha}(z, 0)=1, \\
\lim _{R \rightarrow \pm \infty} W\left(f_{ \pm}\left(z_{ \pm}\right), \psi_{ \pm, \alpha}(z)\right)(R)=0, \quad z \in \mathbb{C} \backslash \mathbb{R}
\end{gathered}
$$


(the latter condition being superfluous at $+\infty$ and/or $-\infty$, i.e., automatically fulfilled if $\tau$ is l.p. at $+\infty$ and/or $-\infty)$. Existence and uniqueness of $\psi_{ \pm, \alpha}(z, x)$ follows from Theorem XIII.2.32 in [10]; they admit the representation

$$
\psi_{ \pm, \alpha}(z, x)=\theta_{\alpha}(z, x)+m_{ \pm, \alpha}(z) \phi_{\alpha}(z, x)
$$

in terms of the Weyl $m$-functions $m_{ \pm, \alpha}(z)$. With our conventions

$$
\begin{gathered}
\pm m_{ \pm, \alpha}(z) \text { is Herglotz, } \quad \pm \operatorname{Im}\left[m_{ \pm, \alpha}(z)\right]>0, \quad \pm z \in \mathbb{C}_{+}, \\
\overline{m_{ \pm, \alpha}(z)}=m_{ \pm, \alpha}(\bar{z}), \quad z \in \mathbb{C} \backslash \mathbb{R}, \\
W\left(\psi_{+, \alpha}(z), \psi_{-, \alpha}(z)\right)=m_{-, \alpha}(z)-m_{+, \alpha}(z) .
\end{gathered}
$$

Moreover, we recall the following facts:

$$
\begin{gathered}
\pm \lim _{\epsilon \downarrow 0} i \epsilon m_{ \pm, \alpha}(\lambda+i \epsilon)= \begin{cases}0, & \phi_{\alpha}(\lambda, \cdot) \notin L^{2}\left(\mathbb{R}_{ \pm}\right), \\
-\left\|\phi_{\alpha}(\lambda, \cdot)\right\|_{2}^{-2}, & \phi_{\alpha}(\lambda, \cdot) \in L^{2}\left(\mathbb{R}_{ \pm}\right), \lambda \in \mathbb{R},\end{cases} \\
m_{ \pm, \alpha_{1}}(z)=\frac{-\sin \left(\alpha_{1}-\alpha_{2}\right)+\cos \left(\alpha_{1}-\alpha_{2}\right) m_{ \pm, \alpha_{2}}(z)}{\cos \left(\alpha_{1}-\alpha_{2}\right)+\sin \left(\alpha_{1}-\alpha_{2}\right) m_{ \pm, \alpha_{2}}(z)}
\end{gathered}
$$

$m_{ \pm, \alpha}(z) \underset{z \rightarrow i \infty}{=} \cot (\alpha) \pm \frac{i}{\sin ^{2}(\alpha)} z^{-1 / 2}-\frac{\cos (\alpha)}{\sin ^{3}(\alpha)} z^{-1}+o\left(z^{-1}\right), \quad \alpha \in(0, \pi)$

$$
m_{ \pm, 0}(z) \underset{z \rightarrow i \infty}{=} \pm i z^{1 / 2}+o(1)
$$

$$
\begin{aligned}
m_{ \pm, \alpha}(z) & =a_{ \pm, \alpha} \pm \int_{\mathbb{R}}\left[\frac{1}{\lambda-z}-\frac{\lambda}{1+\lambda^{2}}\right] d \rho_{ \pm, \alpha}(\lambda), \quad \alpha \in[0, \pi), \\
& =\cot (\alpha) \pm \int_{\mathbb{R}}(\lambda-z)^{-1} d \rho_{ \pm, \alpha}(\lambda), \quad \alpha \in(0, \pi),
\end{aligned}
$$

with

$$
\begin{gathered}
\int_{\mathbb{R}} \frac{d \rho_{ \pm, \alpha}(\lambda)}{1+|\lambda|} \begin{cases}<\infty, & \alpha \in(0, \pi), \\
=\infty, & \alpha=0,\end{cases} \\
\pm \int_{0}^{ \pm \infty} d x \psi_{ \pm, \alpha}\left(z_{1}, x\right) \psi_{ \pm, \alpha}\left(z_{2}, x\right)= \pm \frac{m_{ \pm, \alpha}\left(z_{1}\right)-m_{ \pm, \alpha}\left(z_{2}\right)}{z_{1}-z_{2}} \\
=\int_{\mathbb{R}}\left(\lambda-z_{1}\right)^{-1}\left(\lambda-z_{2}\right)^{-1} d \rho_{ \pm, \alpha}(\lambda) .
\end{gathered}
$$


While the meaning of (A.38) is clear whenever $\tau$ is l.p. at $\pm \infty$, its interpretation in the l.c. case is as follows: Pick an $m_{+, \alpha_{2}}(z)$ (respectively $m_{-, \alpha_{2}}(z)$ ) on the corresponding limit circle of $\tau$ at $+\infty$ (respectively $-\infty$ ) for $\alpha_{2}$. Then the left-hand-side of (A.38) defines a point $m_{+, \alpha_{1}}(z)$ (respectively $\left.m_{-, \alpha_{1}}(z)\right)$ on the corresponding limit circle of $\tau$ at $+\infty$ (respectively $-\infty$ ) for $\alpha_{1}$. As a consequence, a more sophisticated notation for $\psi_{ \pm, \alpha}(z, x), m_{ \pm, \alpha}(z), d \rho_{ \pm, \alpha}(\lambda)$, etc. would have to include an additional subscript $\varphi_{ \pm}(\alpha) \in[0, \pi)$ parametrizing points on the limit circle at $\pm \infty$ for $\alpha$. For simplicity, we decided to omit this additional subscript in the limit circle case.

Perhaps the asymptotic expansions (A.39) and (A.40) also warrant a comment. Under our general hypothesis (A.12), the standard literature usually provides somewhat weaker asymptotic formulas. The actual results (A.39), (A.40) appear to be due to Everitt [11] (see also [3]).

The Green's function $G\left(z, x, x^{\prime}\right)$ of $H$ is then characterized by

$$
\left((H-z)^{-1} f\right)(x)=\int_{\mathbb{R}} d x^{\prime} G\left(z, x, x^{\prime}\right) f\left(x^{\prime}\right), \quad z \in \mathbb{C} \backslash \sigma(H), f \in L^{2}(\mathbb{R}),
$$

$$
G\left(z, x, x^{\prime}\right)=\frac{1}{m_{-, \alpha}(z)-m_{+, \alpha}(z)} \begin{cases}\psi_{-, \alpha}(z, x) \psi_{+, \alpha}\left(z, x^{\prime}\right), & x \leq x^{\prime} \\ \psi_{-, \alpha}\left(z, x^{\prime}\right) \psi_{+, \alpha}(z, x), & x^{\prime} \leq x\end{cases}
$$

Again (cf. the paragraph following (A.27)), for each $x \in \mathbb{R}$, the diagonal Green's function

$$
G(z, x, x) \text { is Herglotz. }
$$

We emphasize that our choice of reference point $x=0$ in (A.16) was purely a matter of convenience. In Section 3 it turns out to be advantageous to introduce a (variable) reference point $x=y$ instead. Without going into further details at this point, we agree to add the subscript $y$ in this case and hence use the notation $\theta_{\alpha, y}(z, x), \phi_{\alpha, y}(z, x), \psi_{ \pm, \alpha, y}(z, x), m_{ \pm, \alpha, y}(z), d \rho_{ \pm, \alpha, y}(\lambda)$, etc. The Weyl $M$-matrix for $H$ is then defined by

\section{(A.48)}

$$
\begin{aligned}
M_{\alpha, y}(z)= & \left(M_{\alpha, y, p, q}(z)\right)_{1 \leq p, q \leq 2} \\
= & {\left[m_{-, \alpha, y}(z)-m_{+, \alpha, y}(z)\right]^{-1} } \\
& \times\left(\begin{array}{cc}
m_{-\alpha, y}(z) m_{+, \alpha, y}(z) \\
{\left[m_{-, \alpha, y}(z)+m_{+, \alpha, y}(z)\right] / 2}
\end{array}\right.
\end{aligned}
$$

By inspection,

$$
\operatorname{det}\left[M_{\alpha, y}(z)\right]=-\frac{1}{4}
$$

and

$$
M_{\alpha, y, p, p}(z) \text { are Herglotz, } \quad p=1,2 .
$$




\section{REFERENCES}

[1] S. Albeverio, F. Gesztesy, R. Høegh-Krohn, and H. Holden, Solvable Models in Quantum Mechanics, Springer, New York, 1988. MR 90a:81021

[2] N. Aronszajn and W.F. Donoghue, On exponential representations of analytic functions in the upper half-plane with positive imaginary part, J. Anal. Math. 5 (1957), 321-388.

[3] F.V. Atkinson, On the location of the Weyl circles, Proc. Roy. Soc. Edinburgh 88A (1981), 345-356. MR 83a:34023

[4] G. Borg, Eine Umkehrung der Sturm-Liouvilleschen Eigenwertaufgabe, Acta Math. 78 (1946), 1-96. MR 7:382d

[5] - Uniqueness theorems in the spectral theory of $y^{\prime \prime}+(\lambda-q(x)) y=0$, Proc. 11th Scandinavian Congress of Mathematicians, Johan Grundt Tanums Forlag, Oslo, 1952, pp. 276-287. MR 15:315a

[6] E.A. Coddington and N. Levinson, Theory of Ordinary Differential Equations, Krieger, Malabar, 1985.

[7] W. Craig, The trace formula for Schrödinger operators on the line, Commun. Math. Phys. 126 (1989), 379-407. MR 90m:47063

[8] P. Deift and E. Trubowitz, Inverse scattering on the line, Commun. Pure Appl. Math. 32 (1979), 121-251. MR 80e:34011

[9] B.A. Dubrovin, Periodic problems for the Korteweg-de Vries equation in the class of finite band potentials, Funct. Anal. Appl. 9 (1975), 215-223. MR 58:6480

[10] N. Dunford and J.T. Schwartz, Linear Operators, Part II. Spectral Theory, Wiley, New York, 1988. MR 90g:47001b

[11] W.N. Everitt, On a property of the $m$-coefficient of a second-order linear differential equation, J. London Math. Soc. 4 (1972), 443-457. MR 45:7156

[12] H. Flaschka, On the inverse problem for Hill's operator, Arch. Rat. Mech. Anal. 59 (1975), 293-309. MR 52:8550

[13] I.M. Gel'fand and B.M. Levitan, On the determination of a differential equation from its spectral function, Izv. Akad. Nauk SSSR Ser. Mat. 15 (1951), 309-360 (Russian); English transl. in Amer. Math. Soc. Transl. (2) 1 (1955), 253-304. MR 17:489c

[14] F. Gesztesy and H. Holden, On new trace formulae for Schrödinger operators, Acta Applicandae Math. 39 (1995), 315-333.

[15] F. Gesztesy and B. Simon, The xi function, Acta Math. (to appear).

[16] _ Rank one perturbations at infinite coupling, J. Funct. Anal. 128 (1995), 245-252.

[17] F. Gesztesy, H. Holden, and B. Simon, Absolute summability of the trace relation for certain Schrödinger operators, Commun. Math. Phys. 168 (1995), 137-161.

[18] F. Gesztesy, B. Simon, and G. Teschl, work in preparation.

[19] F. Gesztesy, H. Holden, B. Simon, and Z. Zhao, Trace formulae and inverse spectral theory for Schrödinger operators, Bull. Amer. Math. Soc. 29 (1993), 250-255. MR 94c:34127

[20] _ Higher order trace relations for Schrödinger operators, Rev. Math. Phys. (to appear).

[21] H. Grosse and A. Martin, Theory of the inverse problem for confining potentials (I). Zero angular momentum, Nucl. Phys. B148 (1979), 413-432.

[22] H. Hochstadt, On the determination of a Hill's equation from its spectrum, Arch. Rat. Mech. Anal. 19 (1965), 353-362. MR 31:6019

[23] V.A. Javrjan, On the regularized trace of the difference between two singular Sturm-Liouville operators, Soviet Math. Dokl. 7 (1966), 888-891. MR 34:1883

[24] _ A certain inverse problem for Sturm-Liouville operators, Izv. Akad. Nauk Armjan. SSR Ser. Mat. 6 (1971), 246-251. (Russian) MR 46:723

[25] A. Kiselev and B. Simon, Rank one perturbations with infinitesimal coupling, J. Funct. Anal. 130 (1995), 345-356.

[26] S. Kotani and M. Krishna, Almost periodicity of some random potentials, J. Funct. Anal. 78 (1988), 390-405. MR 89i:60133

[27] M.G. Krein, Perturbation determinants and a formula for the traces of unitary and selfadjoint operators, Soviet Math. Dokl. 3 (1962), 707-710.

[28] B.M. Levitan, On the closure of the set of finite-zone potentials, Math. USSR Sbornik 51 (1985), 67-89. MR 25:2446

[29] , Inverse Sturm-Liouville Problems, VNU Science Press, Utrecht, 1987. MR 89b:34001 
[30] B.M. Levitan and M.G. Gasymov, Determination of a differential equation by two of its spectra, Russian Math. Surveys 19:2 (1964), 1-63. MR 29:299

[31] B.M. Levitan and I.S. Sargsjan, Introduction to Spectral Theory, Amer. Math. Soc., Providence, RI, 1975. MR 51:6026

[32] V.A. Marchenko, Some questions in the theory of one-dimensional linear differential operators of the second order, I, Trudy Moskov. Mat. Obšc̆. 1 (1952), 327-420 (Russian); English transl. in Amer. Math. Soc. Transl. (2) 101 (1973), 1-104. MR 15:315

[33] _ Sturm-Liouville Operators and Applications, Birkhäuser, Basel, 1986. MR 88f:34034

[34] H.P. McKean and P. van Moerbeke, The spectrum of Hill's equation, Invent. Math. 30 (1975), 217-274. MR 53:936

[35] H.P. McKean and E. Trubowitz, Hill's operator and hyperelliptic function theory in the presence of infinitely many branch points, Commun. Pure Appl. Math. 29 (1976), 143-226. MR 55:761

[36] D.B. Pearson, Quantum Scattering and Spectral Theory, Academic Press, London, 1988. MR 91k:81198

[37] M. Reed and B. Simon, Methods of Modern Mathematical Physics, II. Fourier Analysis, Self-Adjointness, Academic Press, New York, 1975. MR 58:12429b

[38] B. Simon, Spectral analysis of rank one perturbations and applications, Mathematical Quantum Theory II: Schrödinger Operators (J. Feldman, R. Froese, L.M. Rosen, eds.), Conf. Proc. Canad. Math. Soc., vol. 8, Amer. Math. Soc., Providence, RI, 1995, pp. 109-149.

[39] E. Trubowitz, The inverse problem for periodic potentials, Commun. Pure Appl. Math. 30 (1977), 321-337. MR 55:3408

[40] S. Venakides, The infinite period limit of the inverse formalism for periodic potentials, Commun. Pure Appl. Math. 41 (1988), 3-17. MR 88j:34055

[41] J. Zorbas, Perturbation of self-adjoint operators by Dirac distributions, J. Math. Phys. 21 (1980), 840-847. MR 83b:81035

Department of Mathematics, University of Missouri, Columbia, Missouri 65211

E-mail address: mathfg@mizzou1.missouri.edu

Division of Physics, Mathematics, and Astronomy, California Institute of Technology, 253-37, Pasadena, California 91125 